\title{
A nonlinear equation system approach to the dynamic stochastic user equilibrium simultaneous route and departure time choice problem
}

by

\author{
Jiancheng Long \\ Professor \\ School of Transportation Engineering \\ Hefei University of Technology \\ Hefei 230009, China \\ Tel: +86-551-63831101 \\ E-mail: jianchenglong@hfut.edu.cn
}

\section{W.Y. Szeto}

Assistant Professor

Department of Civil Engineering

The University of Hong Kong

Pokfulam Road, Hong Kong

Tel: +852-28578552

Email: ceszeto@hku.hk

\section{Qin Shi}

Professor

School of Transportation Engineering

Hefei University of Technology

Hefei 230009, China

Tel: +86-551-62919160

E-mail: shiqin@hfut.edu.cn

\section{Ziyou Gao}

Professor

School of Traffic and Transportation

Beijing Jiaotong University

Beijing 100044, China

Tel: +86-10-51688193

E-mail: zygao@bjtu.edu.cn

\section{Hai-Jun Huang}

Professor

School of Economics and Management

Beijing University of Aeronautics and Astronautics

Beijing 100191, China

Tel: +86-10-82316608

E-mail: haijunhuang@buaa.edu.cn 


\begin{abstract}
In dynamic stochastic user equilibrium simultaneous route and departure time choice (DSUE-SRDTC) problems, route travel costs can be non-monotone even if route travel times are monotone with respect to route flows. As a result, the mapping function of the variational inequality (VI) problems for the DSUE-SRDTC problems can be non-monotone, and many existing solution algorithms developed for the DSUE-SRDTC problems do not guarantee convergence under this non-monotone condition. This paper formulates the DSUE-SRDTC problem with fixed demand as a system of nonlinear equations. The mapping function of the proposed system of nonlinear equations is defined by a dynamic route choice problem, which can also be formulated as a VI problem with a strictly monotone mapping function under some assumptions. This property enables that the solution algorithm for the DSUE-SRDTC problem can avoid the requirement of the monotonicitiy of the route travel cost functions for the convergence of the solution procedure. A backtracking inexact Broyden-Fletcher-Goldfarb-Shanno (BFGS) method is adopted to solve the system of nonlinear equations, and iterative methods are developed to generate an initial solution for the BFGS method and solve the dynamic route choice problem. Finally, numerical examples are set up to show that the proposed method outperforms many existing algorithms for solving the DSUE-SRDTC problem in terms of guaranteeing solution convergence.
\end{abstract}

Keywords: Dynamic traffic assignment; Dynamic stochastic user equilibrium; Simultaneous route and departure time choice; Nonlinear equations; Iterative method; BFGS method. 


\section{Introduction}

Dynamic traffic assignment (DTA) problems have two fundamental components: the traffic flow component and the travel choice principle (Szeto and Lo, 2006). The traffic flow component depicts how traffic propagates inside a traffic network and hence governs the network performance in terms of travel time (see e.g., Szeto, 2008; Sumalee et al., 2012; Ngoduy, 2013; Zhang et al., 2013; Zhong et al., 2013; Zhu et al., 2013; Balijepalli et al., 2014; Chen et al., 2014; Chiabaut et al., 2014). This is sometimes referred to as a dynamic network loading (DNL) model.

A travel choice principle models travelers' propensity to travel, e.g., how they select their routes, departure times, modes, or destinations. Traditionally, most of DTA problems assume that travelers have perfect travel information and their travel choices are governed by either the dynamic system optimal principle (e.g., Merchant and Nemhauser, 1978a,b; Ma et al., 2014; Mesa-Arango and Ukkusuri, 2014; Shen and Zhang, 2014) or the dynamic user optimal (DUO) principle (e.g., Iryo, 2013; Blumberg-Nitzani and Bar-Gera, 2014). The DUO principle assumes that travelers select their routes and/or departure times to minimize their actual travel costs. However, travelers may have imperfect information and different perceptions toward travel costs rather than perfect information and homogeneous perceptions (Han, 2003). To overcome this limitation, the dynamic stochastic user equilibrium (DSUE) principle was proposed to model how travelers behave in transportation networks (e.g., Vythoulkas, 1990; Chen and Feng, 2000; Lim and Heydecker, 2005; Szeto et al., 2011). These two principles can be adopted in the three categories of DTA problems (Szeto and Wong, 2012): (1) the pure departure time choice problems (e.g., Vickrey, 1969; Arnott et al., 1990; Lindsey, 2004; Qian and Zhang, 2013; Siu and Lo, 2013), (2) the pure route choice problems (e.g., Merchant and Nemhauser, 1978a,b; Carey and Srinivasan, 1993; Carey and Subrahmanian, 2000; Ziliaskopoulos, 2000; Lo and Szeto, 2002a,b; Han, 2003; Ban et al., 2008, 2012a,b; Chow, 2009; Nie and Zhang, 2010; Mounce and Carey, 2011; Nie, 2011; Zheng and Chang, 2011; Carey and Ge, 2012; Carey and Watling, 2012; Han et al., 2013a; Long et al., 2013; Zhu and Ukkusuri, 2013), and (3) the simultaneous route and departure time choice (SRDTC) problems (e.g., Mahmassani and Herman, 1984; Friesz et al., 1993; Ran and Boyce, 1996; Huang and Lam, 2002; Szeto and Lo, 2004; Heydecker and Addison, 2005; Lim and Heydecker, 2005; Mun, 2011; Han et al., 2013b; Siu and Lo, 2013; Friesz and Meimand, 2014). This paper mainly focuses on the DSUE SRDTC (DSUE-SRDTC) problem with fixed demand.

Similar to the DSUE route choice problems (e.g., Vythoulkas, 1990; Szeto et al., 2011, Meng and Khoo, 2012), the DSUE-SRDTC problems can be formulated as fixed point (FP) problems (e.g., Lim and Heydecker, 2005), i.e., multiplying OD demands with route and departure choice probabilities 
yields route flows, where the choice probabilities are functions of route flows. The DSUE-based DTA problems formulated as FP problems are usually solved by the method of successive averages (MSA) (e.g., Ran and Boyce, 1996; Han, 2003), which relies on a predetermined stepsize for guaranteeing convergence. However, the convergence speed of the MSA is slow due to improper stepsize (i.e., too large or too small for some iterations). Liu et al. (2009) proposed a self-regulated averaging method (SRAM) to deal with the slow convergence problem and to solve the deterministic-network SUE problem. SRAM was further used by Szeto et al. (2011) to solve a multi-class doubly stochastic dynamic traffic assignment. If the stepsize in the MSA is fixed to be 1.0, then it becomes the pure network loading (PNL) method (Han, 2003). Nevertheless, these solution algorithms cannot always guarantee convergence even for the DSUE route choice problems (see examples in Han, 2003 for details).

The DSUE-SRDTC problems can also be analytically formulated as variational inequality (VI) problems (e.g., Chen, 1999), and be solved by any general computational technique developed for VI problems, such as the MSA (e.g., Ran and Boyce, 1996; Ran et al., 2002), the diagonalisation method (e.g., Ran and Boyce, 1996; Han, 2003), the projection method (e.g., Wu et al., 1998; Chen and Feng, 2000; Zhang et al., 2001), and so on, provided that the convergence requirements are satisfied. The convergence of solution algorithms for VI problems is mainly determined by the continuity and monotonicity of their mapping functions. For example, the convergence of projection-based method developed for VI problems requires their mapping functions to be continuous and strictly monotone (Theorem 1.6 in Nagurney, 1993; Jang et al., 2005). This strictly monotone requirement is in fact stronger than necessary and other solution algorithms for VI problems with weaker convergence requirements were used in the literature. For instance, the convergence of both the alternating direction method (Lo and Szeto, 2002a,b) and the descent method (Szeto and Lo, 2004) requires the mapping function of the VI problems to be co-coercive. Co-coercive mappings are monotone and Lipschitz continuous but may not necessarily be strongly monotone (Zhao and Hu, 2007). Many algorithms with even weaker convergence requirements have been developed to solve DTA problems formulated as VI problems, such as the day-to-day route swapping algorithm (Huang and Lam, 2002; Szeto and Lo, 2006; Tian et al., 2012) and the extragradient method (Long et al., 2013), which, respectively, require that the mapping functions to be continuous and monotone (Mounce and Carey, 2011) and to be Lipschitz continuous and pseudomonotone (Panicucci et al., 2007) for guaranteeing convergence. In summary, the convergence of solution methods for VI problems generally requires that the mapping functions are (Lipschitz) continuous and (strictly or pseudo-) monotone. However, we found that the generalized route travel cost can be non-monotone with respect to route flows, even if the travel time function is monotone. This leads to that the mapping function of the VI 
problem for the DSUE-SRDTC problem can be non-monotone, and the convergence requirement of many existing solution algorithms for the DSUE-SRDTC problems which are formulated as VI problems in general networks cannot be met.

Different from traditional methods, this paper analytically reformulates the DSUE-SRDTC problem with fixed demand as a system of nonlinear equations instead of a FP problem or a VI problem, whose decision variables are not route flows but the minimum perceived travel costs of OD pairs instead. Under the DSUE-SRDTC condition, the perceived travel costs are the same for all travelers who belong to the same OD pair. If the minimum perceived travel costs are given for all OD pairs, then the total fixed demand for each OD pair can be retrieved endogenously by the specified equilibrium cost via solving a relaxed DSUE-SRDTC problem. In this case, we can solve the relaxed DSUE-SRDTC problem to obtain the equilibrium route flows and the corresponding total OD demands, which are the sum of route flows associated with the OD pairs over time. By setting the retrieved OD demands equal to the actual OD demands, we can define a system of nonlinear equations, whose mapping function is defined by a relaxed DSUE-SRDTC problem. We found that the relaxed DSUE-SRDTC problem can be equivalently reformulated as a dynamic route choice problem. Both the relaxed DSUE-SRDTC problem and the equivalent dynamic route choice problem can be formulated as a VI problem. However, the mapping function of the VI problem for the former can be non-monotone, while the mapping function of the VI problem for the latter is strictly monotone under some assumptions. Therefore, we can solve the equivalent dynamic route choice problem instead of the relaxed DSUE-SRDTC problem to evaluate the mapping function of the proposed system of nonlinear equations.

This paper develops an iterative method to solve the VI problem for the DSUE-SRDTC problem and the VI problem for the equivalent dynamic route choice problem of the relaxed DSUE-SRDTC problem. As mentioned above, the mapping function of the VI problem for the DSUE-SRDTC problem can be non-monotone, and the proposed iterative method may fail to solve the DSUE-SRDTC problem. However, the mapping function of the VI problem for the dynamic route choice problem is strictly monotone under some assumptions, and hence the iterative method guarantees convergence if the assumptions are satisfied. This paper also adopts the Broyden-Fletcher-Goldfarb-Shanno (BFGS) method, which is one of the most popular members of quasi-Newton methods, to solve the proposed system of nonlinear equations. The advantage of a BFGS algorithm over a Newton method is that the super-linear convergence of the former is sufficiently fast, while its computational complexity is significantly less than that of the Newton method. The major difficulty for solving the system of nonlinear equations by the BFGS methods is the lack of practical line search strategy. A backtracking inexact BFGS method proposed by Yuan and Lu (2008) is adopted to solve the proposed system of 
nonlinear equations. The merits of this method include the following: It has a norm descent property (i.e., the search direction is descent for the norm function), and its global and superlinear convergence can be achieved under mild conditions (Yuan and Lu, 2008). Compared with traditional methods for solving the DSUE-SRDTC problem, the proposed method can avoid the requirement of the monotonicitiy of the route travel cost functions for the convergence of the solution procedure. It only requires the route travel time functions are monotone. Numerical experiments show that the proposed method outperforms many existing solution algorithms in terms of guaranteeing convergence.

The contributions of this paper include the following:

First, this paper provides a new approach to formulate the DSUE-SRDTC problem. Instead of using route flows as decision variables as in the classical VI or FP formulations, equilibrium cost is used as a decision variable in a system of nonlinear equations. This formulation approach can be adopted to formulate other DTA or traffic assignment problems.

Second, we develop a novel, efficient, and robust solution methodology to solve the DSUE-SRDTC problem with fixed demand. This methodology relies on the backtracking inexact BFGS method to determine OD travel cost and an iterative scheme to solve many DSUE route choice subproblems and determine an initial feasible solution. The convergence of our algorithm only requires the monotonicity of route travel time.

Third, we illustrate the non-monotonicity of generalized route travel cost functions in DSUE-SRDTC problems. This implies that most of existing algorithms that rely on the monotonicity of the mapping function of VI may not be able to get optimal solutions for DSUE-SRDTC problems.

The rest of this paper is organized as follows: In the next section, the FP and VI formulations of the DSUE-SRDTC problem is presented, and an alternative DSUE-SRDTC condition is developed. The DSUE-SRDTC problem is equivalently reformulated as a system of nonlinear equations in Section 3. Section 4 provides a BFGS method to solve the DSUE-SRDTC problem. Numerical examples are given in Section 5 to illustrate the performance of the proposed solution algorithms. Finally, conclusions are drawn in Section 6.

\section{Dynamic stochastic user equilibrium simultaneous route and departure time choice problem}

\subsection{Notations}

We consider a network $G(N, A)$ with multiple origins and destinations, where $N$ is defined as the set of nodes and $A$ denotes the set of arcs (links). $R$ and $S$ denote the set of origin nodes and the set of destination nodes, respectively. We discretize the time period $T$ of interest into a finite set of time intervals $K=\{k=1,2, \cdots, \underline{K}\}$. Let $\delta$ be the interval length such that $\delta \underline{K}=T$. The demand period $\left[0, T_{d}\right]$ is assumed to be long enough, and travelers depart from their origins at a set of time 
intervals $K_{d}=\left\{k=1,2, \cdots, \underline{K}_{d}\right\}$, where $\underline{K}_{d}<\underline{K}$ and $\delta \underline{K}_{d}=T_{d}$. The following notations are adopted throughout this paper:
$P^{r s} \quad$ set of routes connecting OD pair $(r, s)$.
$f_{p}^{r s}(k) \quad$ flow entering route $p \in P^{r s}$ during interval $k$.
f vector of route flows ( $\left.f_{p}^{r s}(k), \forall r \in R, s \in S, p \in P^{r s}, k \in K_{d}\right)$.
$Q^{r s} \quad$ total demand for OD pair $(r, s)$ during the demand period.
Q vector of total demands $\left(Q^{r s}, \forall r \in R, s \in S\right)$.
$q^{r s}(k) \quad$ traffic demand OD pair $(r, s)$ during interval $k$.
$t_{p}^{r s}(k) \quad$ actual route travel time for flow entering route $p \in P^{r s}$ during interval $k$. It is a function of route flows $\mathbf{f}$
$\psi_{k}^{r s}(t) \quad$ generalized travel cost function with respect to the travel time $t$ for travelers departing from origin $r$ to destination $s$ during interval $k$, which is assumed to be continuous and strictly increasing.
$\psi_{k}^{r s-1}(\cdot) \quad$ inverse function of $\psi_{k}^{r s}(t)$.
$c_{p}^{r s}(k) \quad$ generalized travel cost incurred by travelers entering route $p \in P^{r s}$ during interval $k$ and $c_{p}^{r s}(k)=\psi_{k}^{r s}\left(t_{p}^{r s}(k)\right)$.
c vector of generalized travel costs $\left(c_{p}^{r s}(k), \forall r \in R, s \in S, p \in P^{r s}, k \in K_{d}\right)$.
$\hat{c}_{p}^{r s}(k) \quad$ generalized travel cost perceived by travelers entering route $p \in P^{r s}$ during interval $k$.
c vector of perceived generalized travel costs $\left(\hat{c}_{p}^{r s}(k), \forall r \in R, s \in S, p \in P^{r s}, k \in K_{d}\right)$.
$\bar{C}^{r s}(k) \quad$ expected perceived travel cost for travelers who depart from origin $r$ and travel to destination $s$ during interval $k$.
$\hat{\pi}^{r s} \quad$ minimum perceived travel cost between OD pair $(r, s)$ during the studied period.
$\hat{\boldsymbol{\pi}} \quad$ vector of minimum perceived travel costs $\left(\hat{\pi}^{r s}, \forall r \in R, s \in S\right)$.
$P^{r s}(k) \quad$ probability of travelers who depart from origin $r$ during interval $k$ and travel to destination $s$.
$P_{p}^{r s}(k) \quad$ probability of travelers entering route $p \in P^{r s}$ during interval $k$.
$\Omega \quad$ feasible solution set of the DSUE-SRDTC problem.

\subsection{Overview of the DSUE-SRDTC problem}

Following the static stochastic user equilibrium principle (Daganzo and Sheffi, 1977), the DSUE-SRDTC condition can be stated as follows: In a dynamic stochastic user equilibrium network, no traveler can improve his/her perceived travel cost by unilaterally changing his/her departure time and route combination. The DSUE-SRDTC problem assumes that the perceived travel costs of travelers are imprecise. To model the perceived travel cost, an error term is often added to the actual 
travel cost, and many probability distributions have been applied to model the error. The perceived route travel cost for travelers equals then the sum of two components, a systematic term and an error term, formulated as follows:

$$
\hat{c}_{p}^{r s}(k)=c_{p}^{r s}(k)+\varepsilon_{p}^{r s}(k),
$$

where $\varepsilon_{p}^{r s}(k)$ is the random component of the travel cost of route $p \in P^{r s}$ perceived by travelers, which is determined by the probability distributions of experienced route travel cost. If $\varepsilon_{p}^{r s}(k)$ is assumed to be independently and identically distributed Gumbel variates, then a logit-based choice model is resulted:

$$
P_{p}^{r s}(k)=\frac{\exp \left(-\theta_{r} c_{p}^{r s}(k)\right)}{\sum_{l \in P^{r s}} \exp \left(-\theta_{r} c_{l}^{r s}(k)\right)},
$$

where $\theta_{r}$ is a dispersion parameter associated with traveler's route choice.

For the logit model, travelers' expected perceived travel cost can be formulated as follows (Williams, 1977; Lim and Heydecker, 2005):

$$
\bar{c}^{r s}(k)=E\left[\min _{k \in P^{r s}}\left\{\hat{c}_{k}^{r s}(k)\right\}\right]=-\frac{1}{\theta_{r}} \ln \sum_{l \in P^{r s}} \exp \left(-\theta_{r} c_{l}^{r s}(k)\right) .
$$

Similar to the dynamic route choice model, if the expected travel cost associated with the departure time is assumed to be independently and identically distributed Gumbel variates, then we can obtain a logit-based departure time choice model:

$$
P^{r s}(k)=\frac{\exp \left(-\theta_{t} \bar{c}^{r s}(k)\right)}{\sum_{h \in K_{d}} \exp \left(-\theta_{t} \bar{c}^{r s}(h)\right)},
$$

where $\theta_{t}$ is a dispersion parameter associated with traveler's departure time choice, and $\theta_{t} \leq \theta_{r}$ (Lim and Heydecker, 2005).

By definition, we have

$$
\begin{aligned}
& q^{r s}(k)=Q^{r s} P^{r s}(k), \text { and } \\
& f_{p}^{r s}(k)=q^{r s}(k) P_{p}^{r s}(k) .
\end{aligned}
$$

Eqs. (5) and (6) imply flow conservation, i.e., the summation of route flows equals the total OD demand, given as follows:

$$
\sum_{k \in K_{d}} \sum_{p \in P^{r s}} f_{p}^{r s}(k)=Q^{r s}
$$

Eqs. (5) and (6) also imply flow non-negativity:

$$
\mathbf{f} \geq \mathbf{0},
$$

because total demands and route and departure time choice probabilities are non-negative. 
By substituting (2) and (4) into Eqs. (6) and (5) respectively, we have

$$
\begin{aligned}
q^{r s}(k) & =Q^{r s} \frac{\exp \left(-\theta_{t} \bar{c}^{r s}(k)\right)}{\sum_{h \in K_{d}} \exp \left(-\theta_{t} \bar{c}^{r s}(h)\right)}, \text { and } \\
f_{p}^{r s}(k) & =q^{r s}(k) \cdot \frac{\exp \left(-\theta_{r} c_{p}^{r s}(k)\right)}{\sum_{l \in P^{r s}} \exp \left(-\theta_{r} c_{l}^{r s}(k)\right)} .
\end{aligned}
$$

Lim and Heydecker (2005) provided a DSUE condition for the logit-based SRDTC problem, which can be used to check the solution quality of the DSUE-SRDTC problem. This paper develops an alternative DSUE condition for the logit-based SRDTC problem, given as follows

Theorem 1. (DSUE-SRDTC condition). Let $f_{p}^{r s}(k) \geq 0$ be flow that enters route $p \in P^{r s}$ during interval $k$ and is calculated according to the logit-based choice model. Then, there exists a vector $\boldsymbol{\pi}^{*}=\left[\pi^{r s^{*}}\right]$ such that the following condition is satisfied:

$$
f_{p}^{r s}(k)>0 \Rightarrow \pi_{1}^{r s}(k)+\pi_{2}^{r s}(k, p)=\pi^{r s^{*}},
$$

where

$$
\begin{aligned}
& \pi_{1}^{r s}(k)=\left(\frac{1}{\theta_{t}}-\frac{1}{\theta_{r}}\right) \ln \sum_{l \in P^{r s}} f_{l}^{r s}(k), \text { and } \\
& \pi_{2}^{r s}(k, p)=c_{p}^{r s}(k)+\frac{1}{\theta_{r}} \ln f_{p}^{r s}(k) .
\end{aligned}
$$

Proof. Taking logarithms on both sides of Eqs. (9) and (10) and rearranging the resultant equations, we have

$$
\begin{aligned}
& \bar{c}^{r s}(k)+\frac{1}{\theta_{t}} \ln q^{r s}(k)=\frac{1}{\theta_{t}} \ln Q^{r s}-\frac{1}{\theta_{t}} \ln \sum_{h \in K_{d}} \exp \left(-\theta_{t} \bar{c}^{r s}(h)\right), \text { and } \\
& c_{p}^{r s}(k)+\frac{1}{\theta_{r}} \ln f_{p}^{r s}(k)=\frac{1}{\theta_{r}} \ln q^{r s}(k)-\frac{1}{\theta_{r}} \ln \sum_{l \in P^{r s}} \exp \left(-\theta c_{l}^{r s}(k)\right)=\frac{1}{\theta_{r}} \ln q^{r s}(k)+\bar{c}^{r s}(k)
\end{aligned}
$$

Since the right hand side of Eq. (14) is independent of a specific time interval $k$, we can define:

$$
\pi^{r s^{*}}=\frac{1}{\theta_{t}} \ln Q^{r s}-\frac{1}{\theta_{t}} \ln \sum_{h \in K_{d}} \exp \left(-\theta_{t} \bar{c}^{r s}(h)\right) .
$$

Substituting Eq. (16) into Eq. (14) and rearranging the resultant equation, we have

$$
\bar{c}^{r s}(k)=\pi^{r s^{*}}-\frac{1}{\theta_{t}} \ln q^{r s}(k) .
$$

Substituting Eqs. (17) into Eq. (15) and rearranging the resultant equation, we have

$$
c_{p}^{r s}(k)+\left(\frac{1}{\theta_{t}}-\frac{1}{\theta_{r}}\right) \ln \sum_{l \in P^{r s}} f_{l}^{r s}(k)+\frac{1}{\theta_{r}} \ln f_{p}^{r s}(k)=\pi^{r s^{*}} \text {, or }
$$




$$
\pi_{1}^{r s}(k)+\pi_{2}^{r s}(k, p)=\pi^{r s^{*}}
$$

where $\pi_{1}^{r s}(k)$ and $\pi_{2}^{r s}(k, p)$ are expressed by Eqs. (12) and (13), respectively. This completes the proof. $\square$

Theorem 2. (Special case of the DSUE-SRDTC condition). For the special case of $\theta_{t}=\theta_{r}$, there exists a vector $\boldsymbol{\pi}^{*}=\left[\pi^{r s^{*}}\right]$ such that the following condition is satisfied:

$$
f_{p}^{r s}(k)>0 \Rightarrow \pi_{2}^{r s}(k, p)=\pi^{r s^{*}}
$$

where $\pi_{2}^{r s}(k, p)$ is given by Eq. (13).

Proof. Substituting $\theta_{t}=\theta_{r}$ into Eq. (12), we have $\pi_{1}^{r s}(k)=0$. Therefore, Eq. (11) implies

$$
f_{p}^{r s}(k)>0 \Rightarrow \pi_{1}^{r s}(k)+\pi_{2}^{r s}(k, p)=\pi_{2}^{r s}(k, p)=\hat{\pi}^{r s} .
$$

This completes the proof.

\subsection{An equivalent VI formulation}

Similar to the stochastic dynamic route choice problem (Chen and Feng, 2000; Han, 2003), the DSUE-SRDTC problem can be formulated as a VI problem. According to Theorem 1, the DSUE-SRDTC condition can be rewritten as follows:

$$
\hat{c}_{p}^{r s}(k)\left\{\begin{array}{l}
=\hat{\pi}^{r s}, \text { if } f_{p}^{r s}(k)>0, \\
\geq \hat{\pi}^{r s}, \text { if } f_{p}^{r s}(k)=0,
\end{array} \forall r \in R, s \in S, p \in P^{r s}, k \in K_{d},\right.
$$

where $\hat{c}_{p}^{r s}(k)$ is the generalized travel cost perceived by travelers entering route $p \in P^{r s}$ during interval $k$, and $\hat{\pi}^{r s}$ is the minimum perceived generalized travel cost for travelers between OD pair ( $r$, s). They can be formulated as follows:

$$
\begin{aligned}
& \hat{\pi}^{r s}=\min \left[\hat{c}_{p}^{r s}(k)\right] \text {, and } \\
& \hat{c}_{p}^{r s}(k)=\pi_{1}^{r s}(k)+\pi_{2}^{r s}(k, p)=c_{p}^{r s}(k)+\left(\frac{1}{\theta_{t}}-\frac{1}{\theta_{r}}\right) \ln \sum_{l \in P^{r s}} f_{l}^{r s}(k)+\frac{1}{\theta_{r}} \ln f_{p}^{r s}(k) .
\end{aligned}
$$

The first term on the right hand side of the second equality sign in Eq. (21) is the actual route travel cost, and the second and the third terms on the right hand side of the second equality sign in Eq. (21) are perceived components of the perceived travel cost, respectively.

Equivalently, the DSUE-SRDTC condition can be rewritten as follows:

$$
\begin{aligned}
& f_{p}^{r s}(k)\left[\hat{c}_{p}^{r s}(k)-\hat{\pi}^{r s}\right]=0, \forall r \in R, s \in S, p \in P^{r s}, k \in K_{d}, \text { and } \\
& \hat{c}_{p}^{r s}(k)-\hat{\pi}^{r s} \geq 0, \forall r \in R, s \in S, p \in P^{r s}, k \in K_{d} .
\end{aligned}
$$


The logit-based DSUE-SRDTC problem with fixed demand is to determine a vector of route flows to satisfy (7), (8), and (20)-(23). This problem can be expressed as the following VI problem:

Theorem 3. The DSUE-SRDTC problem (7), (8), and (20)-(23) can be equivalently formulated as a finite-dimensional VI problem: finding a vector $\mathbf{f}^{*} \in \Omega$ such that

$$
\sum_{r \in R} \sum_{s \in S} \sum_{p \in P^{r s}} \sum_{k \in K_{d}} \hat{c}_{p}^{r s^{*}}(k)\left[f_{p}^{r s}(k)-f_{p}^{r s^{*}}(k)\right] \geq 0, \forall \mathbf{f} \in \Omega,
$$

where $\Omega$ is a closed convex set, and

$$
\Omega=\left\{\mathbf{f} \geq \mathbf{0}: \sum_{p \in P^{r s}} \sum_{k \in K_{d}} f_{p}^{r s}(k)=Q^{r s}, \forall r \in R, s \in S\right\} .
$$

The proof directly follows Friesz et al. (1993) and Chen (1999).

\subsection{Generalized route travel cost function and its non-monotonicity}

The generalized travel cost for travelers departing from $r$ to $s$ is assumed to be made up of two components (Huang and Lam, 2002; Szeto and Lo, 2004; Lim and Heydecker, 2005): (1) travel time of the trip, and (2) a "penalty" for arriving at destinations earlyllate. We assume that travelers going to their destinations have a desired arrival time period, expressed as the arrival time window $\left[k_{s}^{*}-\Delta_{s}, k_{s}^{*}+\Delta_{s}\right.$, where $k_{s}^{*}$ is the middle point of the time window and may represent the official work start time at destination $s, \Delta_{s}$ is the interval of arrival time flexibility. Travelers will incur schedule delay costs if they arrive at the destination outside this arrival time window; otherwise, they acquire no schedule delay cost for arriving within the desired arrival time window. The following generalized travel cost function is adopted in this paper:

$$
\psi_{k}^{r s}(t)=\alpha t+ \begin{cases}\beta\left[k_{s}^{*}-\Delta_{s}-k-t\right], & \text { if } k+t<k_{s}^{*}-\Delta_{s}, \\ \gamma\left[k+t-k_{s}^{*}-\Delta_{s}\right], & \text { if } k+t>k_{s}^{*}+\Delta_{s}, \\ 0, & \text { otherwise, }\end{cases}
$$

where $\alpha$ is the unit cost of travel time; $\beta$ and $\gamma$ are the unit cost of schedule delay time-early and -late, respectively. According to empirical results (Small, 1982), we have $\beta<\alpha<\gamma$.

Substituting the route travel time into the generalized travel cost function (26), we can obtain the generalized route travel cost as follows:

$$
c_{p}^{r s}(k)=\alpha t_{p}^{r s}(k)+ \begin{cases}\beta\left[k_{s}^{*}-\Delta_{s}-k-t_{p}^{r s}(k)\right], & \text { if } k+t_{p}^{r s}(k)<k_{s}^{*}-\Delta_{s}, \\ \gamma\left[k+t_{p}^{r s}(k)-k_{s}^{*}-\Delta_{s}\right], & \text { if } k+t_{p}^{r s}(k)>k_{s}^{*}+\Delta_{s}, \\ 0, & \text { otherwise. }\end{cases}
$$

Since the DSUE-SRDTC problem is formulated as a VI problem and the solution set is closed and convex, if its mapping function (i.e., perceived route travel cost functions) is continuous with respect to route flows, then there is at least a solution to the problem. If its mapping function is further strictly 
monotone with respect to route flows, then there is exactly one solution to the problem. According to Huang and Lam (2002) and Szeto and Lo (2004), route travel cost functions are continuous if route travel times are continuous with respect to route flows. It is obvious that the perceived components of the perceived route travel costs in Eq. (21) are continuous with respect to route flows. Therefore, the perceived route travel costs are continuous with respect to route flows. This implies that if route travel times are continuous with respect to route flows, then the mapping function of the VI problem for the DSUE-SRDTC problem is continuous and the DSUE-SRDTC problem must have at least a solution.

Smith and Ghali (1990) proved that route travel times are monotonic with respect to the route flows for a single bottleneck. However, Ghali and Smith (1993) pointed out that the generalized route travel cost functions are not necessarily monotone if there are multiple active bottlenecks per route, but they did not give an example to show it. In the following example, we show that the actual route travel cost functions can be non-monotone, even if the route travel time function is monotone.

Example 1. Non-monotonicity of route travel cost functions.

We consider the single bottleneck model (Vickrey, 1969). Assume that there is a highway with a single bottleneck connecting a residential district with the central commercial district (CBD). Without loss of generality, we assume the travel time from bottleneck to destination is zero, and the official work start time is zero. The DUO-based solution to the bottleneck model is given as follows (see Arnott et al., 1990, for details):

$$
r(t)=\left\{\begin{array}{l}
\frac{\alpha}{\alpha-\beta} s, \text { for } t \in\left[t_{s}, t_{o}\right], \\
\frac{\alpha}{\alpha+\gamma} s, \text { for } t \in\left[t_{o}, t_{e}\right] .
\end{array}\right.
$$

where $t_{\mathrm{s}}=-\frac{\gamma}{\beta+\gamma} \frac{N}{\mathrm{~s}}, t_{0}=-\frac{\beta \gamma}{\beta+\gamma} \frac{N}{\alpha \mathrm{s}}, t_{e}=\frac{\beta}{\beta+\gamma} \frac{N}{\mathrm{~s}}$.

Consider a different flow pattern that is resulted from an adjustment of the equilibrium flow pattern. We increase commuters' departure rates during $\left[t_{s}, t_{0}\right]$ by $\Delta_{1}$ and reduce the commuters' departure rates during $\left[t_{s}, t_{0}\right]$ by $\Delta_{2}$. Let $\tilde{r}(t)$ be commuters' departure rates after the adjustment, and $\tilde{\mathbf{r}}=(\tilde{r}(t), \forall t)$. If the total number of commuters is not changed, then we have

$$
\begin{aligned}
& \left(t_{e}-t_{o}\right) \Delta_{2}=\left(t_{o}-t_{s}\right) \Delta_{1}, \text { and } \\
& \tilde{r}(t)=\left\{\begin{array}{l}
\frac{\alpha}{\alpha-\beta} s+\Delta_{1}, \text { for } t \in\left[t_{s}, t_{o}\right], \\
\frac{\alpha}{\alpha+\gamma} s-\Delta_{2}, \text { for } t \in\left[t_{o}, t_{e}\right] .
\end{array}\right.
\end{aligned}
$$


The route flow rates in Eqs. (28) and (30) generate the following dot products (see Appendix A for details):

$$
\begin{aligned}
\langle\tilde{\mathbf{c}}-\mathbf{c}, \tilde{\mathbf{r}}-\mathbf{r}\rangle & =-\frac{\alpha(\beta+\gamma) \Delta_{1}^{2}\left(t_{o}-t_{s}\right)^{2}}{2\left[\alpha s+\Delta_{1}(\alpha-\beta)\right]}, \text { and } \\
\langle\tilde{\hat{\mathbf{c}}}-\hat{\mathbf{c}}, \tilde{\mathbf{r}}-\mathbf{r}\rangle & =-\frac{\alpha(\beta+\gamma) \Delta_{1}^{2}\left(t_{o}-t_{s}\right)^{2}}{2\left[\alpha s+\Delta_{1}(\alpha-\beta)\right]}+\frac{\Delta_{1}\left(t_{o}-t_{s}\right)}{\theta_{t}} \ln \frac{\alpha s+\Delta_{1}(\alpha-\beta)}{\alpha s-\Delta_{1}(\alpha-\beta) \gamma / \beta},
\end{aligned}
$$

where $\mathbf{r}$ is the vector of flow rates, and $\mathbf{r}=(r(t), \forall t)$. $\mathbf{c}$ and $\tilde{\mathbf{c}}$ are the vectors of the actual travel costs before and after the flow adjustment, respectively. $\hat{\mathbf{c}}$ and $\tilde{\hat{\mathbf{c}}}$ are the vectors of the perceived travel costs before and after the flow adjustment, respectively.

Since $\alpha>\beta$ and all parameters on the right hand side of Eq. (31) are positive, the only term on the right hand side of Eq. (31) is negative, i.e., $\langle\tilde{\mathbf{c}}-\mathbf{c}, \tilde{\mathbf{r}}-\mathbf{r}\rangle<0$. This implies that the actual route travel cost function is non-monotone.

We set $\alpha=1.0, \beta=0.5, \gamma=1.5, N=8000$ veh, $s=60 \mathrm{veh} / \mathrm{min}$, and $\Delta_{1}=5 \mathrm{veh} / \mathrm{min}$. Substituting the parameters into Eqs. (28)-(32), we have

$$
\langle\tilde{\hat{\mathbf{c}}}-\hat{\mathbf{c}}, \tilde{\mathbf{r}}-\mathbf{r}\rangle=-1500+\frac{63.39}{\theta_{t}} \text {. }
$$

According to Eq. (33), $(\tilde{\hat{\mathbf{c}}}-\hat{\mathbf{c}})^{\mathrm{T}}(\tilde{\mathbf{r}}-\mathbf{r})<0$ if $\theta_{t}>0.04226 \mathrm{~min}^{-1}$. This implies that the perceived route travel cost function can be non-monotone with respect to route flow rates, even if the route travel time function is monotone. Therefore, the mapping function of the VI problem (24) may be non-monotone due to the non-monotonicity of the actual route travel cost function, and the DSUE-SRDTC problem may not have a unique optimal solution. This property leads to the situation that many existing solution algorithms (e.g., Han, 2003; Lim and Heydecker, 2005; Szeto et al., 2011) developed for solving the DSUE-SRDTC problems cannot guarantee convergence under this non-monotonicity condition.

\section{A novel formulation}

\subsection{A relaxed DSUE-SRDTC problem}

According to Theorem 1, the perceived route travel cost for the used routes are equal and minimal. If the minimum perceived route travel cost of each OD pair is given and the demand constraints are eliminated, then we can obtain a relaxed DSUE-SRDTC problem where its optimal flow pattern also satisfies the DSUE-SRDTC conditions (22) and (23). Similarly, the relaxed DSUE-SRDTC problem can also be formulated as a VI problem: finding a vector $\mathbf{f}^{*} \in R^{+}$such that 


$$
\sum_{k \in K_{d}} \sum_{r \in R} \sum_{s \in S} \sum_{p \in P^{r s}}\left[\hat{c}_{p}^{r r^{*}}(k)-\hat{\pi}^{r s}\right]\left[f_{p}^{r s}(k)-f_{p}^{r s^{*}}(k)\right] \geq 0, \forall \mathbf{f} \in R^{+} .
$$

where $\hat{\pi}^{r s}$ is the minimum perceived route travel cost associated with OD pair $(r, s)$ and is predetermined for the VI problem (34).

The relaxed DSUE-SRDTC problem can be equivalently reformulated as a dynamic route choice problem, given as follows:

Theorem 4. The VI problem (34) can be equivalently formulated as the following VI problem: finding a vector $\mathbf{f}^{*} \in R^{+}$such that

$$
\sum_{k \in K_{d}} \sum_{r \in R} \sum_{s \in S} \sum_{p \in P^{r s}}\left[t_{p}^{r s^{*}}(k)-\eta_{p}^{r s^{*}}(k)\right]\left[f_{p}^{r s}(k)-f_{p}^{r s^{*}}(k)\right] \geq 0, \forall \mathbf{f} \in R^{+} .
$$

where $\eta_{p}^{r s}(k)$ is the reference route travel time, and

$$
\eta_{p}^{r s}(k)=\psi_{k}^{r s-1}\left(\hat{\pi}^{r s}-\left(\frac{1}{\theta_{t}}-\frac{1}{\theta_{r}}\right) \ln \sum_{l} f_{l}^{r s}(k)-\frac{1}{\theta_{r}} \ln f_{p}^{r s}(k)\right) .
$$

Proof. Firstly, we prove that any solutions of the VI problem (35) are also solutions of the VI problem (34). Let $\mathbf{f}^{*} \in R^{+}$be an optimal solution to the VI problem (35). Then, we have

$$
t_{p}^{r s^{*}}(k)\left\{\begin{array}{l}
=\eta_{p}^{r s^{*}}(k), \text { if } f_{p}^{r s^{*}}(k)>0 \\
\geq \eta_{p}^{r s^{*}}(k), \text { if } f_{p}^{r s^{*}}(k)=0
\end{array}\right.
$$

Since the function $\psi_{k}^{r s}(t)$ is monotonic increasing, Eq. (37) implies

$$
\psi_{k}^{r s}\left(t_{p}^{r s^{*}}(k)\right)\left\{\begin{array}{l}
=\psi_{k}^{r s}\left(\eta_{p}^{r s^{*}}(k)\right), \text { if } f_{p}^{r s^{*}}(k)>0, \\
\geq \psi_{k}^{r s}\left(\eta_{p}^{r s^{*}}(k)\right), \text { if } f_{p}^{r s^{*}}(k)=0 .
\end{array}\right.
$$

According to Eqs. (21) and (36), respectively, we have

$$
\begin{aligned}
& \hat{c}_{p}^{r s^{*}}(k)=\psi_{k}^{r s}\left(t_{p}^{r s^{*}}(k)\right)+\left(\frac{1}{\theta_{t}}-\frac{1}{\theta_{r}}\right) \ln \sum_{l} f_{l}^{r s^{*}}(k)+\frac{1}{\theta_{r}} \ln f_{p}^{r s^{*}}(k), \text { and } \\
& \hat{\pi}^{r s}=\psi_{k}^{r s}\left(\eta_{p}^{r s^{*}}(k)\right)+\left(\frac{1}{\theta_{t}}-\frac{1}{\theta_{r}}\right) \ln \sum_{l} f_{l}^{r s^{*}}(k)+\frac{1}{\theta_{r}} \ln f_{p}^{r s^{*}}(k) .
\end{aligned}
$$

Combining Eqs. (38)-(40), we can conclude that $\mathbf{f}^{*}$ satisfies the DSUE-SRDTC condition (19). Hence, $\mathbf{f}^{*}$ is also an optimal solution to the VI problem (34).

Secondly, we prove that any solutions of the VI problem (34) are also solutions of the VI problem (35). Let $\mathbf{f}^{*} \in R^{+}$be an optimal solution to the VI problem (34). Then, $\mathbf{f}^{*}$ must satisfy the DSUE-SRDTC condition (19) and $f_{p}^{r s^{*}}(k)>0$ implies $\hat{c}_{p}^{r s^{*}}(k) \geq \pi^{r s} . f_{p}^{r s^{*}}(k)>0$ further implies $\hat{c}_{p}^{r s^{*}}(k)-\pi^{r s} \geq 0$ and the following

$$
\hat{c}_{p}^{r s^{*}}(k)-\left(\frac{1}{\theta_{t}}-\frac{1}{\theta_{r}}\right) \ln \sum_{l} f_{l}^{r s}(k)-\frac{1}{\theta_{r}} \ln f_{p}^{r s}(k) \geq \hat{\pi}^{r s}-\left(\frac{1}{\theta_{t}}-\frac{1}{\theta_{r}}\right) \ln \sum_{l} f_{l}^{r s}(k)-\frac{1}{\theta_{r}} \ln f_{p}^{r s}(k) .
$$

Since the function $\psi_{k}^{r s}(t)$ is monotonic increasing, its inverse function $\psi_{k}^{r s-1}(\cdot)$ is also monotonic 
increasing. Therefore, we can substitute each side of inequality (41) into the function $\psi_{k}^{r s-1}(\cdot)$ to obtain the condition $t_{p}^{r s^{*}}(k) \geq \eta_{p}^{r s^{*}}(k)$ and obtain the condition that

$$
\text { If } f_{p}^{r s^{*}}(k)>0 \text {, then } \hat{c}_{p}^{r s^{*}}(k)-\hat{\pi}^{r s} \geq 0 \Rightarrow t_{p}^{r s^{*}}(k) \geq \eta_{p}^{r s^{*}}(k) \text {. }
$$

Similarly, we also have the condition that

$$
\text { If } f_{p}^{r s^{*}}(k)=0 \text {, then } \hat{c}_{p}^{r s^{*}}(k)-\hat{\pi}^{r s}=0 \Rightarrow t_{p}^{r s^{*}}(k)=\eta_{p}^{r s^{*}}(k) .
$$

Eqs. (42) and (43) imply that the condition (37) is satisfied. Therefore, $\mathbf{f}^{*} \in R^{+}$is also an optimal solution to the VI problem (35).

Assumption 1. Route travel times are continuous, differentiable and monotone with respect to route flows.

Assumption 2. The matrix $-\frac{1}{2}\left(\nabla_{\mathbf{f}} \boldsymbol{\eta}+\nabla_{\mathbf{f}} \boldsymbol{\eta}^{\mathrm{T}}\right)$ is a strictly diagonally dominant matrix.

Lemma 1. (Johnson, 1970). An $n \times n$ matrix $A$ is positive definite if and only if the symmetric part of $A$ is positive definite, i.e., $B=\frac{1}{2}\left(A+A^{\mathrm{T}}\right)$ is positive definite.

Proposition 1. Under Assumptions 1 and 2, the VI problems (34) and (35) guarantee solution existence and solution uniqueness.

Proof. Since the function $\psi_{k}^{r s}(t)$ is continuous, $\psi_{k}^{r s-1}(\cdot)$ is also a continuous function, and hence Eq. (36) implies that $\boldsymbol{\eta}$ is continuous with respect to route flows. Under Assumption 1, route travel times are continuous with respect to route flows, and hence the mapping function of the VI problem (35) is continuous. The solution set is also convex and can be reduced to a closed set because the flow cannot be greater than its corresponding OD demand. Therefore, the VI problem (35) guarantees solution existence (Theorem 1.4 in Nagurney, 1993).

We can obtain the diagonal elements of the Jacobian matrix $\nabla_{\mathrm{f}} \boldsymbol{\eta}$ as follows:

$$
\begin{aligned}
& \frac{\partial \eta_{p}^{r s}(k)}{\partial f_{p}^{r s}(k)}= \\
& \psi_{k}^{r s-1^{\prime}}\left(\hat{c}^{r s^{*}}-\left(\frac{1}{\theta_{t}}-\frac{1}{\theta_{r}}\right) \ln \sum_{l} f_{l}^{r s}(k)-\frac{1}{\theta_{r}} \ln f_{p}^{r s}(k)\right) \cdot\left[-\left(\frac{1}{\theta_{t}}-\frac{1}{\theta_{r}}\right) \frac{1}{\sum_{l} f_{l}^{r s}(k)}-\frac{1}{\theta_{r} f_{p}^{r s}(k)}\right] .
\end{aligned}
$$

Since the function $\psi_{k}^{r s}(t)$ is strictly increasing, its inverse function $\psi_{k}^{r s-1}(\cdot)$ is also strictly increasing, and the derivative of $\psi_{k}^{r s-1}(\cdot)$ is positive. Since $\theta_{t} \leq \theta_{r}$ and $f_{p}^{r s}(k)$ is positive, the diagonal elements of the Jacobian matrix $\nabla_{\mathbf{f}} \boldsymbol{\eta}$ are negative. Hence, the diagonal elements of the matrix $-\frac{1}{2}\left(\nabla_{\mathbf{f}} \boldsymbol{\eta}+\nabla_{\mathbf{f}} \boldsymbol{\eta}^{\mathrm{T}}\right)$ are positive. Moreover, under Assumption 2, $-\frac{1}{2}\left(\nabla_{\mathbf{f}} \boldsymbol{\eta}+\nabla_{\mathbf{f}} \boldsymbol{\eta}^{\mathrm{T}}\right)$ is a strictly diagonally dominant matrix. Hence, the matrix is positive definite. According to Lemma 1 , $-\nabla_{\mathbf{f}} \boldsymbol{\eta}$ is positive definite, and hence $-\boldsymbol{\eta}$ is strictly monotone with respect to route flows. Under Assumption 1, route travel times are monotone with respect to route flows, and hence the mapping 
function of the VI problem (35) is strictly monotone with respect to route flows. This and the condition for solution existence imply that the VI problem (35) guarantees solution uniqueness (Theorem 1.6 in Nagurney, 1993).

According to Theorem 4, the VI problem (35) is equivalent with the VI problem (34). Hence, the VI problem (34) also guarantees solution existence and solution uniqueness.

The non-diagonal elements of $-\nabla_{\mathbf{f}} \boldsymbol{\eta}^{\mathrm{T}}$ are given as follows:

$$
\begin{aligned}
& -\frac{\partial \eta_{p}^{r s}(k)}{\partial f_{l}^{r s}(k)}= \\
& \varphi_{k}^{r s-1^{\prime}}\left(\tilde{c}^{r s^{*}}-\left(\frac{1}{\theta_{t}}-\frac{1}{\theta_{r}}\right) \ln \sum_{l} f_{l}^{r s}(k)-\frac{1}{\theta_{r}} \ln f_{p}^{r s}(k)\right) \cdot\left[\left(\frac{1}{\theta_{t}}-\frac{1}{\theta_{r}}\right) \frac{1}{\sum_{l} f_{l}^{r s}(k)}\right], \forall l \neq p
\end{aligned}
$$

Since the function $\psi_{k}^{r s}(t)$ is strictly increasing, its inverse function $\psi_{k}^{r s-1}(\cdot)$ is also strictly increasing, and the derivative of $\psi_{k}^{r s-1}(\cdot)$ is positive. Since $\theta_{t} \leq \theta_{r}$ and $f_{p}^{r s}(k)$ is positive, the non-diagonal elements of $-\nabla_{\mathbf{f}} \boldsymbol{\eta}^{\mathrm{T}}$ are positive. When the value of $\theta_{t}$ is closer to the value of $\theta_{r}$, the values of the non-diagonal elements of $-\nabla_{\mathbf{f}} \boldsymbol{\eta}^{\mathrm{T}}$ are smaller, and $-\frac{1}{2}\left(\nabla_{\mathbf{f}} \boldsymbol{\eta}+\nabla_{\mathbf{f}} \boldsymbol{\eta}^{\mathrm{T}}\right)$ is more likely to be a diagonally dominant matrix.

Proposition 2. Under Assumption 1, if $\theta_{t}=\theta_{r}$, then the VI problems (34) and (35) guarantee solution existence and solution uniqueness.

Proof. If $\theta_{t}=\theta_{r}$, then we have

$$
\begin{aligned}
& \frac{\partial \eta_{p}^{r s}(k)}{\partial f_{p}^{r s}(k)}=-\psi_{k}^{r s-1^{\prime}}\left(\hat{c}^{r s^{*}}-\frac{1}{\theta_{r}} \ln f_{p}^{r s}(k)\right) \cdot \frac{1}{\theta_{r} f_{p}^{r s}(k)} \text {, and } \\
& \frac{\partial \eta_{p}^{r s}(k)}{\partial f_{l}^{r s}(k)}=0, l \neq p .
\end{aligned}
$$

Eqs. (44) and (45) imply that $-\nabla_{\mathbf{f}} \boldsymbol{\eta}$ is a diagonal matrix with positive diagonal elements, and Assumption 2 is satisfied, i.e., the matrix $-\frac{1}{2}\left(\nabla_{\mathbf{f}} \boldsymbol{\eta}+\nabla_{\mathbf{f}} \boldsymbol{\eta}^{\mathrm{T}}\right)$ is a strictly diagonally dominant matrix. According to Proposition 1, the VI problems (34) and (35) guarantee solution existence and solution uniqueness under Assumption 1. This completes the proof.

In the VI problem (34), the vector of the minimum perceived OD travel costs $\hat{\boldsymbol{\pi}}$ is a model input. For any given $\hat{\boldsymbol{\pi}}$, we can obtain an optimal solution to the VI problem (34). Using Eq. (7), we can further retrieve the corresponding vector of OD demands. Therefore, the vector of retrieved OD demands is a function of the vector of the minimum perceived OD travel costs $\hat{\boldsymbol{\pi}}$, denoted by $\mathbf{S}(\hat{\boldsymbol{\pi}})$ and named the retrieved OD demand function. According to Proposition 2, the optimal solution to the VI problems (34) and (35) is unique, and hence we have the following corollaries: 
Corollary 1. Under Assumptions 1 and 2, the function $\mathbf{S}(\hat{\boldsymbol{\pi}})$ is an injective function.

Corollary 2. Under Assumption 1, if $\theta_{t}=\theta_{r}$, then the function $\mathbf{S}(\hat{\boldsymbol{\pi}})$ is an injective function.

\subsection{The DSUE-SRDTC problem formulated as a system of nonlinear equations}

According to the preceding discussion, the vector of retrieved OD demands is a function of the vector of the minimum perceived OD travel costs $\hat{\boldsymbol{\pi}}$. Let the vector function of retrieved OD demands be equal to the vector of total demands, i.e., $\mathbf{S}(\hat{\boldsymbol{\pi}})=\mathbf{Q}$. Then, we can obtain a system of nonlinear equations, whose decision variables are the minimum generalized perceived OD travel costs $\hat{\boldsymbol{\pi}}$. The value of retrieved OD demand function $\mathbf{S}(\hat{\boldsymbol{\pi}})$ can be obtained by solving a relaxed DSUE-SRDTC problem (34), while the total demand vector $\mathbf{Q}$ is an input to the DSUE-SRDTC problem and is predetermined. In order to simplify the description of the solution algorithm in the next section, we define the following system of nonlinear equations:

$$
\mathbf{Z}(\hat{\boldsymbol{\pi}})=\mathbf{S}(\hat{\boldsymbol{\pi}})-\mathbf{Q}=\mathbf{0} .
$$

Theorem 5. Under Assumptions 1 and 2, for any solutions of nonlinear equations (46), the corresponding route flow solution is the unique optimal solution to the VI problem (24).

Proof. Let $\hat{\boldsymbol{\pi}}^{*}$ be a solution to the system of nonlinear equations (46) and $\mathbf{f}^{*} \in R^{+}$be the optimal solution to the VI problem (34) with $\hat{\boldsymbol{\pi}}=\hat{\boldsymbol{\pi}}^{*}$. This optimal route flow solution is unique according to Proposition 1 under Assumptions 1 and 2. Since the optimal solution to the VI problem (34) must satisfy the DSUE-SRDTC conditions (22) and (23), we have

$$
\begin{aligned}
& f_{p}^{r s^{*}}(k)\left[\hat{c}_{p}^{r s^{*}}(k)-\hat{\pi}^{r s^{*}}\right]=0, \forall r \in R, s \in S, p \in P^{r s}, k \in K_{d}, \text { and } \\
& c_{p}^{r s^{*}}(k)-\hat{\pi}^{r s^{*}} \geq 0, \forall r \in R, s \in S, p \in P^{r s}, k \in K_{d} .
\end{aligned}
$$

Let $\mathbf{Q}^{*}$ be the vector of retrieved OD demands, i.e., $\sum_{p \in P^{r s}} \sum_{k \in K_{d}} f_{p}^{r s^{*}}(k)=Q^{r s^{*}}$. By the definition of retrieved OD demand functions, we have $\mathbf{S}\left(\hat{\boldsymbol{\pi}}^{*}\right)=\mathbf{Q}^{*}$. Since $\hat{\boldsymbol{\pi}}^{*}$ is a solution to the system of nonlinear equations (46), we have

$$
\mathbf{S}\left(\hat{\boldsymbol{\pi}}^{*}\right)=\mathbf{Q} \text {. }
$$

Hence, we have

$$
\sum_{p \in P^{r s}} \sum_{k \in K_{d}} f_{p}^{r s^{*}}(k)=Q^{r s^{*}}=Q^{r s}
$$

Since $\mathbf{f}^{*} \in R^{+}$satisfies Eqs. (47), (48), and (50), $\mathbf{f}^{*}$ is an optimal solution to DSUE-SRDTC problem. It is also a unique optimal solution to the VI problem (24) according to Theorem 3.

Theorem 6. Under Assumption 1, if $\theta_{t}=\theta_{r}$, then for any solutions of nonlinear equations (46) the corresponding route flow solution is an optimal solution to the VI problem (24).

The proof is similar to that of Theorem 5 . 
Assumption 3. The function $\mathbf{S}(\hat{\boldsymbol{\pi}})$ is monotone.

Proposition 3. Under Assumption 3, $\mathbf{Z}(\hat{\boldsymbol{\pi}})$ is monotone.

Proof. Since the traffic demand $\mathbf{Q}$ is assumed to be fixed in this paper, $-\mathbf{Q}$ is monotone with respect to $\hat{\boldsymbol{\pi}}$. Under Assumption 3, $\mathbf{S}(\hat{\boldsymbol{\pi}})$ is monotone. Therefore, $\mathbf{Z}(\hat{\boldsymbol{\pi}})=\mathbf{S}(\hat{\boldsymbol{\pi}})-\mathbf{Q}$ is monotone with respect to $\hat{\boldsymbol{\pi}}$. This completes the proof.

\section{Solution algorithms}

According to Theorems 5 and 6, we can obtain an optimal solution to the DSUE-SRDTC problem by solving the system of nonlinear equations (46) and the VI problem (35). In this paper, the BFGS method will be adopted to solve the systems of nonlinear equations (46). During the implementation of the BFGS method, we evaluate the mapping function $\mathbf{Z}(\hat{\boldsymbol{\pi}})$, in which the function $\mathbf{S}(\hat{\boldsymbol{\pi}})$ can be obtained by solving the VI problem (35). In this section, we will firstly develop an iterative method to solve the VI problem (24), which can generate an initial solution for the BFGS method. The iterative method will be further extended to solve the VI problem (35). Therefore, the BFGS method forms the main solution algorithm, and the iterative method forms a sub-algorithm. Before describing of the two solution methods, some gap functions are provided to evaluate the convergence of the algorithms used in this paper and the quality of the solutions obtained.

\subsection{Gap functions}

In this paper, the following two gap functions are adopted to evaluate the quality of the solutions of the dynamic route choice problem and the DSUE-SRDTC problem, respectively:

$$
\begin{aligned}
& G_{1}(\mathbf{f})=\max \left\{\left|t_{p}^{r s}(k)-\eta_{p}^{r s}(k)\right|\right\}, \text { and } \\
& G_{2}(\mathbf{f})=\max \left\{\left|\hat{c}_{p}^{r s}(k)-\hat{\pi}^{r s}\right|\right\},
\end{aligned}
$$

Eqs. (51) and (52) give the maximum gap (i.e., the largest difference between the route travel times and the corresponding reference route travel times) for the dynamic route choice problem and the maximum gap (i.e., the largest difference between the route travel costs of all used routes and the corresponding minimum route travel costs) for the DSUE-SRDTC problem, respectively.

The following gap function is used to evaluate the convergence of the BFGS method for the proposed system of nonlinear equations:

$$
G_{3}(\hat{\boldsymbol{\pi}})=\|\mathbf{Z}(\hat{\boldsymbol{\pi}})\| \text {. }
$$

If $\hat{\boldsymbol{\pi}}$ is a solution to nonlinear equations (46), then the gap function (53) is equal to zero and $\hat{\boldsymbol{\pi}}$ is the vector of minimum perceived OD travel costs. Otherwise, the gap is positive.

In order to compare with existing solution methods, the following gap function is adopted to evaluate solution quality for the DSUE-SRDTC problem: 


$$
G_{4}(\mathbf{f})=\sum_{r} \sum_{s} \sum_{p} \sum_{k}\left|Q^{r s} P^{r s}(k) P_{p}^{r s}(k)-f_{p}^{r s}(k)\right| .
$$

According to Eq. (6), if $\mathbf{f}$ is an optimal solution to the DSUE-SRDTC problem, then the gap function (54) is equal to zero. Otherwise, the gap is positive.

\subsection{Iterative method}

We firstly provide the general iterative scheme proposed by Nagurney (1993), and then develop a new smooth function for the general iterative scheme to solve the DSUE-SRDTC problem. The proposed function satisfies the required properties of the generalized iterative scheme.

\subsubsection{General iterative scheme}

Assume that there exists a smooth function

$$
\mathbf{g}(\mathbf{f}, \mathbf{h}): \Omega \times \Omega \mapsto R^{n},
$$

with the following two properties: (i) $\mathbf{g}(\mathbf{f}, \mathbf{f})=\hat{\mathbf{c}}(\mathbf{f})$ for all $\mathbf{f} \in \Omega$, and (ii) for every fixed $\mathbf{f}, \mathbf{h} \in \Omega$, the $n \times n$ matrix,$\nabla_{\mathbf{f}} \mathbf{g}(\mathbf{f}, \mathbf{h})$ is symmetric and positive definite. Then, any function $\mathbf{g}(\mathbf{f}, \mathbf{h})$ with the above properties can be used in the following general iterative solution scheme for the VI problem (24):

Step 0: Initialization. Select an initial solution $\mathbf{f}_{0} \in \Omega$ and set $\imath=0$.

Step 1: Constructing and computing subproblem. Compute $\mathbf{f}_{t+1}$ by solving the following VI problem:

$$
\left\langle\mathbf{g}\left(\mathbf{f}_{t+1}, \mathbf{f}_{l}\right)^{\mathrm{T}}, \mathbf{f}-\mathbf{f}_{t+1}\right\rangle \geq 0, \forall \mathbf{f} \in \Omega .
$$

Step 2. Convergence checking. If $G_{2}\left(\mathbf{f}_{t+1}\right)<\varepsilon_{1}$, where $\varepsilon_{1}$ is a positive convergence tolerance, then stop the algorithm; Otherwise, set $l=\imath+1$, and return to Step 1 .

Since $\nabla_{\mathbf{f}} \mathbf{g}(\mathbf{f}, \mathbf{h})$ is symmetric and positive definite, the line integral $\int \mathbf{g}(\mathbf{f}, \mathbf{h}) d \mathbf{f}$ defines a function $G(\mathbf{f}, \mathbf{h}): \Omega \times \Omega \mapsto R$ such that for a fixed $\mathbf{h} \in \Omega, G(\cdot, \mathbf{h})$ is strictly convex and

$$
\mathbf{g}(\mathbf{f}, \mathbf{h})=\nabla_{\mathbf{f}} G(\mathbf{f}, \mathbf{h}) .
$$

Therefore, the VI problem (56) is equivalent to the following strictly convex mathematical programming problem

$$
\min _{\mathbf{f} \in \Omega} G\left(\mathbf{f}, \mathbf{f}_{l}\right),
$$

in which a unique solution $\mathbf{f}_{t+1}$ exists.

\subsubsection{The proposed smooth function for the iterative scheme}

In the framework of the general iterative scheme, we propose the following smooth function for solving the DSUE-SRDTC problem (24) and the dynamic route choice problem (35):

$$
\mathbf{g}(\mathbf{f}, \mathbf{h})=\mathbf{F}(\mathbf{h})+\frac{1}{\rho}(\ln \mathbf{f}-\ln \mathbf{h}),
$$


where $\rho$ is positive and fixed. It is obvious that function (59) has the two properties required for the general iterative scheme, and we can obtain the line integral of $\mathbf{g}(\mathbf{f}, \mathbf{h})$, given as follows:

$$
G(\mathbf{f}, \mathbf{h})=\int \mathbf{g}(\mathbf{f}, \mathbf{h}) d \mathbf{f}=\frac{1}{\rho}\left[\mathbf{f}^{\mathrm{T}} \ln \mathbf{f}+(\rho \mathbf{F}(\mathbf{h})-\ln \mathbf{h}-\mathbf{e})^{\mathrm{T}} \mathbf{f}\right] .
$$

At each iteration $\imath$, substituting $\mathbf{h}=\mathbf{f}_{\imath}$ into Eq. (60), we can reformulate the subproblem (58) as follows:

$$
\min _{\mathbf{f} \in \Omega} \mathbf{f}^{\mathrm{T}} \ln \mathbf{f}+\left(\rho \mathbf{F}\left(\mathbf{f}_{\imath}\right)-\ln \mathbf{f}_{\imath}-\mathbf{e}\right)^{\mathrm{T}} \mathbf{f} .
$$

where $\mathbf{e}=(1,1, \cdots, 1)^{\mathrm{T}}$. Note that $1 / \rho$ is a positive constant in Eq. (60) and can be ignored in problem (61) without changing the optimal solution.

The Hessian matrix of the objective function of the optimization problem (61) is diagonal, and hence the optimization problem (61) can be decomposed into many small-size problems. For each OD pair $(r, s)$, solve the following problem:

$$
\begin{aligned}
& \min \sum_{p \in P^{r s}} \sum_{k \in K_{d}}\left\{f_{p}^{r s}(k) \ln f_{p}^{r s}(k)+\left[\rho \hat{c}_{p}^{r s}(k)_{\imath}-\ln f_{p}^{r s}(k)_{l}-1\right] f_{p}^{r s}(k)\right\} \\
& \text { s.t. }\left\{\begin{array}{l}
\sum_{p \in P^{r s s}} \sum_{k \in K_{d}} f_{p}^{r s}(k)=Q^{r s}, \\
f_{p}^{r s}(k) \geq 0, \forall r \in R, s \in S, p \in P^{r s}, k \in K_{d} .
\end{array}\right.
\end{aligned}
$$

Since the flows on all routes in the path set are positive for the logit-based DSUE problems, the non-negativity constraints of problem (62) will never be active and hence can be eliminated. Selecting a reference path index $\bar{p} \in P^{r s}$ and a reference interval index $\bar{k} \in K_{d}$, we have

$$
f_{\bar{p}}^{r s}(\bar{k})=Q^{r s}-\sum_{(p, k) \neq(\bar{p}, \bar{k})} f_{p}^{r s}(k) .
$$

Substituting Eq. (63) into the objective function of problem (62), we can reformulate the optimization problem (62) as an unconstrained optimization problem, given as follows:

$$
\begin{aligned}
\min Z= & \left(Q^{r s}-\sum_{(p, k) \neq(\bar{p}, \bar{k})} f_{p}^{r s}(k)\right) \ln \left(Q^{r s}-\sum_{(p, k) \neq(\bar{p}, \bar{k})} f_{p}^{r s}(k)\right)+ \\
& \sum_{(p, k) \neq(\bar{p}, \bar{k})}\left\{f_{p}^{r s}(k) \ln f_{p}^{r s}(k)+\alpha_{p}^{r s}(k) f_{p}^{r s}(k)\right\}+\alpha_{l}^{r s}(h)\left(Q^{r s}-\sum_{(p, k) \neq(\bar{p}, \bar{k})} f_{p}^{r s}(k)\right),
\end{aligned}
$$

where $\alpha_{p}^{r s}(k)$ is fixed for problem (64), and $\alpha_{p}^{r s}(k)=\rho \hat{c}_{p}^{r s}(k)_{t}-\ln f_{p}^{r s}(k)_{t}-1$.

At optimality, the gradient of the unconstrained problem (64) equals zero. Therefore, we have

$$
\frac{\partial Z}{\partial f_{p}^{r s}(k)}=\alpha_{p}^{r s}(k)+\ln f_{p}^{r s}(k)-\alpha_{\bar{p}}^{r s}(\bar{k})-\ln f_{\bar{p}}^{r s}(\bar{k})=0 .
$$

Eq. (65) implies that there exists a positive $\mu^{*}$ such that $\alpha_{p}^{r s}(k)+\ln f_{p}^{r s}(k)=\mu^{*}$ is satisfied for all combinations $(p, k)$ at optimality. Hence, we have 


$$
f_{p}^{r s}(k)=\exp \left\{\mu^{*}-\alpha_{p}^{r s}(k)\right\}
$$

Substituting Eq. (66) in to Eq. (7), we have

$$
\sum_{p \in P^{r s}} \sum_{k \in K_{d}} f_{p}^{r s}(k)=\sum_{p \in P^{r s}} \sum_{k \in K_{d}} \exp \left\{\mu^{*}-\alpha_{p}^{r s}(k)\right\}=Q^{r s} .
$$

The value of $\mu^{*}$ can be obtained by solving the one-dimensional nonlinear equation (67), and can be further substituted into Eq. (66) to obtain the solution to problem (62). In this paper, the one-dimensional nonlinear equation (67) is solved by Newton's method, and the gap function (52) is adopted to check convergence of the iterative method for solving the DSUE-SRDTC problem.

Function (59) was also adopted to develop an iterative method to solve the VI problem (35) and obtain the corresponding subproblem (61). This subproblem can be further decomposed into many small-size problems. For each OD pair $(r, s)$ and each path at each time interval $k$, the sub-algorithm solves the following problem:

$$
\begin{aligned}
& \min f_{p}^{r s}(k) \ln f_{p}^{r s}(k)+\left\{\bar{\rho}\left[t_{p}^{r s}(k)_{l}-\eta_{p}^{r s}(k)_{l}\right]-\ln f_{p}^{r s}(k)_{\iota}-1\right\} f_{p}^{r s}(k) \\
& \text { s.t. } f_{p}^{r s}(k) \geq 0 .
\end{aligned}
$$

where $\bar{\rho}$ is a positive constant.

Solving the optimization problem (68), we have

$$
f_{p}^{r s}(k)_{l+1}=\exp \left\{\ln f_{p}^{r s}(k)_{l}-\bar{\rho}\left[t_{p}^{r s}(k)_{l}-\eta_{p}^{r s}(k)_{l}\right]\right\} .
$$

To check the convergence of the iterative method for solving the DSUE-SRDTC problem (24) and the dynamic route choice problem (35), the gap function (51) was adopted. In theory, the convergence of the iterative scheme for solving these two problems requires their mapping functions to be continuous and strictly monotone (Theorem 1.6 in Nagurney, 1993). Example 1 shows that the mapping function of the VI problem (24) may be non-monotone, and hence the iterative scheme for solving the DSUE-SRDTC problem cannot always guarantee convergence. Propositions 1 and 2 show that the mapping function of the dynamic route choice problem (35) is continuous and strictly monotone under some assumptions, and hence the iterative scheme for solving the dynamic route choice problem guarantees convergence under such assumptions.

\subsection{A BFGS method for solving the DSUE-SRDTC problem}

According to Theorems 5 and 6, we can obtain an optimal solution to the DSUE-SRDTC problem by solving the system of nonlinear equations (46). In this section, a BFGS method is adopted to solve the system of nonlinear equations (46). During the implementation of BFGS method, we evaluate the mapping function $\mathbf{Z}(\hat{\boldsymbol{\pi}})$, in which the function $\mathbf{S}(\hat{\boldsymbol{\pi}})$ can be obtained by solving the VI problem (35) using the iterative method described in Section 4.2.2. Therefore, the BFGS method forms the main solution algorithm, and the iterative method forms a sub-algorithm. 
The BFGS method for solving the system of nonlinear equations (46) is outlined as follows (Yuan and Lu, 2008):

Step 0. Initialization. Select an initial solution $\hat{\boldsymbol{\pi}}_{0}$, an initial symmetric positive definite matrix $\mathbf{H}_{0}$, constants $\mu, \omega \in(0,1)$, the convergence tolerance $\varepsilon_{2}>0$, and let $l=0$.

Step 1. Checking stopping criterion. If $G_{3}\left(\hat{\pi}_{l}\right)<\varepsilon_{2}$, then terminates the algorithm.

Step 2. Determination of the descent directions and stepsize. Obtain the descent direction $\mathbf{d}_{t}=-\mathbf{H}_{t} \mathbf{Z}\left(\hat{\boldsymbol{\pi}}_{t}\right)$. Determine $i_{t}$ be the smallest nonnegative integer $i$ such that

$$
\left\|\mathbf{Z}\left(\hat{\boldsymbol{\pi}}_{l}+\omega^{i} \mathbf{d}_{l}\right)\right\|^{2} \leq\left\|\mathbf{Z}\left(\hat{\boldsymbol{\pi}}_{l}\right)\right\|^{2}+\mu \omega^{i} \mathbf{Z}\left(\hat{\boldsymbol{\pi}}_{l}\right)^{\mathrm{T}} \mathbf{d}_{l} .
$$

Set $\lambda_{l}=\omega^{i_{l}}$.

Step 3. Update of the minimum perceived generalized travel cost. Set $\hat{\boldsymbol{\pi}}_{l+1}=\hat{\boldsymbol{\pi}}_{t}+\lambda_{l} \mathbf{d}_{l}$.

Step 4. Update of the symmetric positive definite matrix. Set $\mathbf{s}_{t}=\hat{\boldsymbol{\pi}}_{t+1}-\hat{\boldsymbol{\pi}}_{t}=\lambda_{t} \mathbf{d}_{t}$, and $\mathbf{y}_{t}=\mathbf{Z}\left(\hat{\boldsymbol{\pi}}_{t+1}\right)-\mathbf{Z}\left(\hat{\boldsymbol{\pi}}_{l}\right)$. If $\mathbf{s}_{l}^{T} \mathbf{y}_{\imath}>0$, then set

$$
\mathbf{H}_{\imath+1}=\mathbf{H}_{\imath}+\frac{\left(\mathbf{s}_{l}^{T} \mathbf{y}_{l}+\mathbf{y}_{l}^{T} \mathbf{H}_{l} \mathbf{y}_{l}\right)\left(\mathbf{s}_{\imath} \mathbf{s}_{l}^{T}\right)}{\left(\mathbf{s}_{l}^{T} \mathbf{y}_{l}\right)^{2}}-\frac{\mathbf{H}_{l} \mathbf{y}_{l} \mathbf{s}_{l}^{T}+\mathbf{s}_{\imath} \mathbf{y}_{l}^{T} \mathbf{H}_{l}}{\mathbf{s}_{l}^{T} \mathbf{y}_{l}} .
$$

Otherwise, let $\mathbf{H}_{t+1}=\mathbf{H}_{t}$. Set $\boldsymbol{l}=\boldsymbol{l}+1$, and return to Step 1 .

In Step 0, the initial solution $\hat{\boldsymbol{\pi}}_{0}$ can be any positive vector. In our numerical example, the iterative method is adopted to generate the initial solution $\hat{\boldsymbol{\pi}}_{0}$. We firstly implement the iterative method until either the value of the gap function $G_{4}$ is less than a predetermined convergence tolerance $\varepsilon_{3}$ or the average value of the gap function $G_{2}$ for consecutive 100 iterations is not decreasing. If the iterative method terminates due to the fact that the first condition is met, we have obtained an approximate solution to the DSUE-SRDTC problem, and the BFGS method will not be implemented. Otherwise, we set the average perceived travel cost of each OD pair as the initial solution of the BFSG method. Following the literature, we set the initial symmetric positive definite matrix $\mathbf{H}_{0}$ to be an identity matrix. Then, the convergence criterion is checked in Step 1.

In Step 2, a backtracking inexact line search technique, which was originally proposed by Yuan and $\mathrm{Lu}$ (2008), is adopted to determine the stepsize. Different from some traditional line search techniques, the technique (70) can avoid the computation of the Jacobian matrix of $\mathbf{Z}(\hat{\boldsymbol{\pi}})$, and thus can reduce the computation difficulty, especially for the large-scale problems. The sub-algorithm in the next subsection is adopted to evaluate the function $\mathbf{S}(\hat{\boldsymbol{\pi}})$ and consequently evaluate the mapping function $\mathbf{Z}(\hat{\boldsymbol{\pi}})$ in Step 2 and also in Step 4. Step 3 updates the minimum perceived generalized travel cost and Step 4 updates the symmetric positive definite matrix.

The advantage of the proposed solution algorithm (which incorporates BFGS as the main algorithm) for the DSUE-SRDTC problem is that it can avoid the requirement of the monotonicitiy of 
the route travel cost functions for the convergence of the solution procedure. Many existing solution algorithms for the DSUE-SRDTC problems require the mapping function of the VI problem for the DSUE-SRDTC problem to be monotone. However, this requirement cannot be met, and many existing solution algorithms may fail to solve the DSUE-SRDTC problems (See numerical examples for details).

The BFGS method has a norm descent property, and its global and superlinear convergence have been proved under mild conditions (Yuan and Lu, 2008):

- $\mathbf{Z}(\hat{\boldsymbol{\pi}})$ is continuously differentiable on an open convex set containing the level set defined by $\Omega=\left\{\hat{\boldsymbol{\pi}}_{l} \mid\left\|\mathbf{Z}\left(\hat{\boldsymbol{\pi}}_{l}\right)\right\| \leq\left\|\mathbf{Z}\left(\hat{\boldsymbol{\pi}}_{0}\right)\right\|\right\}$. The Jacobian of $\mathbf{Z}(\hat{\boldsymbol{\pi}})$ is symmetric, bounded, and positive definite on the convex set.

- $\mathbf{H}_{l}$ is a good approximation to $\mathbf{Z}^{\prime}\left(\hat{\boldsymbol{\pi}}_{t}\right)$.

- $\quad \mathbf{Z}^{\prime}\left(\hat{\boldsymbol{\pi}}_{l}\right)$ is Hölder continuous at an optimal solution.

However, the Jacobian matrix of $\mathbf{Z}(\hat{\boldsymbol{\pi}})$ is in general asymmetric, and hence the global and superlinear convergence of the above BFGS method for the DSUE-SRDTC problem is left for future investigation.

Note that $-\frac{1}{2}\left(\nabla_{\mathbf{f}} \boldsymbol{\eta}+\nabla_{\mathbf{f}} \boldsymbol{\eta}^{\mathrm{T}}\right)$ is a diagonally dominant matrix is a sufficient but not necessary condition for guaranteeing the monotonicity of the mapping function. We did not guarantee this condition to be satisfied in the application of the solution method. If this condition is satisfied, an optimal solution can be obtained by starting with any initial solution. If not, the proposed solution method may not guarantee convergence and can only be considered a heuristic. In such case, we may need to repeatedly use the method with different initial solutions until we obtain an optimal solution.

According to Proposition 3, the mapping function of the proposed system of nonlinear equations is monotone under Assumption 3. Therefore, some other BFGS methods developed for nonlinear monotone equations (e.g., Solodov and Svaiter, 1998; Zhou and Li, 2008) can also be adopted to solve the DSUE-SRDTC problem. The BFGS method developed by Zhou and Li (2008), denoted as M-BFGS, converges globally if the mapping function of the equations is monotone and Lipschitz continuous. Their method does not require the differentiability property of the equation. The global convergence of their method is independent of any merit function. For comparison, the M-BFGS method developed by Zhou and Li (2008) was also adopted to solve the DSUE-SRDTC problem, and the detailed algorithm is presented in Appendix B.

\section{Numerical example}

A great many dynamic network loading (DNL) models can be used to depict how traffic propagates inside a traffic network along assigned routes (see Mun, 2007 for a comprehensive review) 
and hence governs the network performance in terms of travel time. The proposed DTA model is formulated in a general form, and any type of DNL models can be used to estimate the traffic pattern over time, and then calculate link travel times by link travel time models. The DNL model used in this paper is the point-queue (PQ) model (see Huang and Lam, 2002; Nie and Zhang, 2005 for details). The underlying reasons for choosing the PQ model are: (i) the PQ model is easy to calibrate since its parameters, including free-flow travel time and bottleneck capacity, are all well-defined physical quantities that are relatively easy to measure; (ii) the PQ model has an advantage of the computational efficiency, and behaves identically as Daganzo's (1995) cell transmission model (CTM) if queue spillback does not occur (Nie and Zhang, 2005); and (iii) the path travel time and path travel cost obtained from the PQ model are continuous with respect to path flows (Huang and Lam, 2002; Szeto and Lo, 2006). The calculation of route travel time directly follows the method in the study by Huang and Lam (2002). The following example is developed to illustrate the performance of the proposed method for solving the DSUE-SRDTC problem.

Example 2. Sioux Falls network: Comparing the efficiency of the proposed algorithm with existing algorithms

As shown in Fig. 1, the Sioux Falls network is adopted to illustrate the performance of the proposed methods. The Sioux Falls network consists of 24 nodes and 76 links (Leblanc, 1975). Following Han (2003), we considered only 12 OD pairs in the network, and adopted Dial's (1971) STOCH method to generate the reasonable path set under the free-flow condition. The OD pairs and the number of paths in the reasonable path set are given in Table 1. The free flow link travel times and outflow capacities of all links are the same as those in Han (2003). Unless stated otherwise, the total trip demands for all OD pairs are 200 veh, and the parameters for the generalized travel cost function are: $\alpha=1.0, \beta=0.5$, and $\gamma=1.5$. The desired arrival time is $1500 \mathrm{~s}$, and the arrival time flexibility is $100 \mathrm{~s}$. The length of each time interval is $10 \mathrm{~s}$. The parameters of the iterative method for the DSUE-SRDTC problem and the dynamic route choice problem are $\rho=0.01$ and $\bar{\rho}=0.05$, respectively. The parameters for the BFGS method are as follows: $\varepsilon_{1}=1.0 \times 10^{-5}, \varepsilon_{2}=1.0 \times 10^{-2}$ veh, $\mu=0.4$, and $\omega=0.9$. The parameters for the M-BFGS method are as follows: $\sigma=0.1$, $\varpi=0.01$. The Manhattan norm (1-norm) is adopted for the gap function $G_{3}(\hat{\boldsymbol{\pi}})$. The gap function $G_{4}(\mathbf{f})$ was adopted to evaluate the efficiency of the solution algorithms. If the value of this gap function was achieved less than $\varepsilon_{3}=1.0 \times 10^{-4}$, then the solution algorithm was terminated.

Usually, CPU time is widely used to evaluate the efficiency of solution algorithms. However, CPU time relies on the compiler, the coding skills, the performance of the used computer, and so on. Since the implementation of DNL is the most time consuming step in solving DTA models, we mainly use the number of DNLs performed instead of CPU time to evaluate the performance of the solution 
methods tested in this study. We set the maximum number of DNLs performed be 400000 and compared the performance of the proposed methods with three existing solution algorithms: PNL (see Han, 2003 for details), MSA, and SRAM (See Liu et al., 2009 for details). We also adopted IM to represent the iterative method described in Section 4.2, BFGS to represent the backtracking inexact BFGS method (Yuan and Lu, 2008), and M-BFGS to represent the BFGS method for nonlinear monotone equations (Zhou and Li, 2008) for short.

The value of the dispersion parameters plays a significant role on the computation time required by the solution algorithms for the logit-based SUE traffic assignment (e.g., Han, 2003; Bekhor and Toledo, 2005; Long et al., 2010). Firstly, the special case of the DSUE-SRDTC problem, i.e., the DSUE-SRDTC problem with $\theta_{r}=\theta_{t}$, was considered. We changed the value of the dispersion parameter $\theta_{r}$ from $0.05 \mathrm{~s}^{-1}$ to $0.75 \mathrm{~s}^{-1}$, and solved the DSUE-SRDTC problem by six solution algorithms. Table 2 reports the number of DNLs required for each solution algorithm. The results presented in Table 2 show that the number of DNLs required is in general increasing for each solution algorithm as the value of the dispersion parameter $\theta_{r}$ grows up, and the first four solution algorithms (i.e., PNL, MSA, RAM, and IM) can fail to solve the DSUE-SRDTC problem. On the contrary, both the BFGS method and the M-BFGS method can solve the DSUE-SRDTC problem for all the settings of the values of the dispersion parameter $\theta_{r}$ shown in the table, but the BFGS method is much more efficient than the M-BFGS method when the value of the dispersion parameter $\theta_{r}$ grows up. We can also observe from Table 2 that the SRAM outperforms the PNL method and the MSA, and the IM outperforms the SRAM, in terms of the applicable range of $\theta_{r}$.

We were also interested in studying the effect of the value of the dispersion parameter $\theta_{t}$ on the efficiency of the solution algorithms. We fixed $\theta_{r}=1.0 \mathrm{~s}^{-1}$, changed the value of the dispersion parameter $\theta_{t}$ from $0.05 \mathrm{~s}^{-1}$ to $1.00 \mathrm{~s}^{-1}$, and solved the DSUE-SRDTC problem by all six solution algorithms. The number of DNLs required for each solution algorithm is reported in Table 3. One can observe the following from the table. First, the PNL method fails to solve the DSUE-SRDTC problem for all cases. Second, the MSA converges very slow and only can solve the DSUE-SRDTC problem when $\theta_{r} \leq 0.10 \mathrm{~s}^{-1}$ within 400000 DNLs. Third, the SRAM is very efficient when the value of $\theta_{t}$ is very small and becomes inefficient when $\theta_{t} \geq 0.3$. Fourth, the iterative method can solve more cases than that of the SRAM. Fifth, the two BFGS methods are efficient for all cases. The results presented in Table 3 imply that the smaller the value of $\theta_{t}$ is, the easier the DSUE-SRDTC problem can be solved.

We set higher values of dispersion parameters and implemented the BFGS method to solve the DSUE-SRDTC problem. The values of convergence indicators are presented in Fig. 2. From this figure, we can observe that the values of the gap functions quickly decrease as the number of iterations 
grows up. This implies that the BFGS method can solve the DSUE-SRDTC problems with very large values of dispersion parameters. The results presented in Fig. 2 are consistent with those in Tables 2 and 3, i.e., the lower the value of dispersion parameters, the easier the DSUE-SRDTC problem can be solved.

The congestion level is also an important factor that can influence the efficiency of the solution algorithms for the DSUE-SRDTC problem. To illustrate this effect, we changed the OD demand of all OD pairs from 20 veh to 400 veh, and implemented all solution algorithms, respectively. The numbers of DNLs required for all solution algorithms are provided in Table 4. We can observe that the lower the level of congestion, the easier the DSUE-SRDTC problem can be solved. The results presented in Table 4 also show that the iterative method outperforms the three existing methods (i.e., PNL, MSA, and SRAM), and the BFGS method outperforms all other solution algorithms. This is consistent with the results presented in Tables 2 and 3.

According to the first term on the right hand side of Eq. (32), the parameters $\beta$ and $\gamma$ (i.e., the unit cost of schedule delay time-early and the unit cost of schedule time-late) are the main sources for the non-monotonicity property of the perceived travel cost function. If the values of $\beta$ and $\gamma$ are reduced, then the DSUE-SRDTC problem is more likely to be strictly monotone, and hence can be solved easier. Since their effects on the performance of the algorithm are similar, we only chose one of them in the sensitivity analysis. We changed the value of $\gamma$ from 1.0 to 3.0, set $\theta_{r}=\theta_{t}=1.0 \mathrm{~s}^{-1}$, and implemented all solution algorithms, respectively. The number of DNLs required for each solution algorithm is reported in Table 5. We can observe from the table that the three existing solution algorithms (i.e., PNL, MSA, and SRAM) fail to solve the DSUE-SRDTC problem. Moreover, the iterative method can only solve the DSUE-SRDTC problem when the value of $\gamma$ is very small, and both the BFGS method and the M-BFGS method can solve the DSUE-SRDTC problem with all the settings of the values of $\gamma$ shown in the table. The results presented in Table 5 also confirm that the smaller the value of $\gamma$ is, the easier the DSUE-SRDTC problem can be solved.

The DSUE-SRDTC condition and the special case of the DSUE-SRDTC condition in Theorems 1 and 2 state that the perceived travel costs are the same for all travelers of the same OD pair. We set $\theta_{r}=\theta_{t}=0.2 \mathrm{~s}^{-1}$, and solved the DSUE-SRDTC problem by the SRAM, the iterative method and the BFGS method, respectively. The values of $\pi_{1}^{r s}(k)+\pi_{2}^{r s}(k, p)$ with respect to OD pair 6->15 are displayed in Fig. 3. We can observe that the solution obtained by the SRAM does not satisfy the DSUE-SRDTC condition. In other words, not all the values of $\pi_{1}^{r s}(k)+\pi_{2}^{r s}(k, p)$ are the same, but the solutions obtained by the iterative method and the BFGS method agree with the DSUE-SRDTC conditions well. We also provide the route flow rates obtained by the three solution algorithms in Fig. 4. One can observe from the figure that the route flow rates obtained by the SRAM do not agree with 
those obtained by other three methods (i.e., IM, BFGS and M-BFGS) when departure time is greater than 1200 s. Although the absolute differences of the route flow rates obtained by different solution algorithms are very small, the solutions obtained by the proposed method are indeed better than those obtained by the other two existing methods.

\section{Conclusion}

The DSUE-SRDTC problems cannot always be solved by many existing solution algorithms due to the non-monotonicity of route travel cost function. This paper formulates the DSUE-SRDTC problem with fixed demand as a system of nonlinear equations, whose mapping function is defined by a dynamic route choice problem. Since the dynamic route choice problem can be formulated as a VI problem with a strictly monotone mapping function under some assumptions, we can avoid the requirement of monotonicitiy of the route travel cost function for the convergence of the solution algorithm. We develop an iterative method to solve the dynamic route choice problem and adopt a BFGS method to solve the proposed system of nonlinear equations. The initial solution was found by the iterative scheme with a different smooth function. A numerical example was developed to illustrate the performance of the proposed method. The results show that many existing solution algorithms for the DSUE-SRDTC problem cannot converge when the values of dispersion parameters or traffic demand levels are high. On the contrary, the proposed method is convergent under all the settings of the dispersion parameter values and traffic demand levels, and obtains solutions that satisfy DSUE-SRDTC conditions.

In this paper, the PQ model was adopted as the DNL model. This type of models ignores the interactions of adjacent links in a network under heavy congestion such as queue spillovers. This greatly simplifies the modeling framework but at the same time may be not really realistic. Some physical queue models, such as the CTM (Daganzo, 1994, 1995) and the link transmission model (LTM) (Yperman, 2007), can capture important and realistic traffic dynamics such as queue spillbacks that often occur in congested urban traffic networks. However, spillbacks may introduce discontinuities to DTA problems (Szeto and Lo, 2006), and Assumption 1 may not be satisfied. However, once Assumptions 1 and 2 are satisfied by DTA problems with physical queues, the proposed algorithm can be used to obtain their solutions. For future studies, we will develop more efficient solution algorithms, and apply the DTA model to evaluate the effect of various traffic management and transport planning measures such as road pricing (Lo and Szeto, 2005), network design (e.g., Szeto et al., 2010, 2014; Miandoabchi et al., 2012a,b), staggered work hours (e.g., Yushimito et al., 2014), incident detection (e.g., Ghosh and Smith, 2014), and traffic flow/density forecasting (e.g., Szeto et al., 2009; Ye et al., 2012; Anand et al., 2014; Chiou et al., 2014). 


\section{Acknowledgements}

This work is jointly supported the National Natural Science Foundation of China (71271075, 71431003, 71271183), the Fok Ying Tung Education Foundation (141081), the Program for New Century Excellent Talents in University (NCET-13-0766), a grant from the Research Grants Council of the Hong Kong Special Administrative Region, China (HKU 17207214E), and a grant (201311159123) from the University Research Committee of the University of Hong Kong. The authors are grateful to the three reviewers for their constructive comments.

\section{Appendix A}

In the single bottleneck model, the queue length obeys the following dynamic equation (Yang and Huang, 2005):

$$
\frac{d q(t)}{d t}=r(t)-s, \text { for } q(t)>0 .
$$

Under the equilibrium condition, the queue length and the travel time are given as follows:

$$
\begin{aligned}
& q(t)=\left\{\begin{array}{l}
\frac{\beta}{\alpha-\beta} s\left(t-t_{s}\right), \text { for } t \in\left[t_{s}, t_{o}\right], \\
\frac{\beta}{\alpha-\beta} s\left(t_{o}-t_{s}\right)-\frac{\gamma}{\alpha+\gamma} s\left(t-t_{o}\right), \text { for } t \in\left[t_{o}, t_{e}\right],
\end{array}\right. \text { and } \\
& T(t)=\frac{q(t)}{s}=\left\{\begin{array}{l}
\frac{\beta}{\alpha-\beta}\left(t-t_{s}\right), \text { for } t \in\left[t_{s}, t_{o}\right], \\
\frac{-\gamma}{\alpha+\gamma}\left(t-t_{o}\right)+\frac{\beta}{\alpha-\beta}\left(t_{o}-t_{s}\right), \text { for } t \in\left[t_{o}, t_{e}\right] .
\end{array}\right.
\end{aligned}
$$

The linear travel cost function is given as follows:

$$
C(t)=\left\{\begin{array}{l}
\alpha \frac{q(t)}{s}+\beta\left[t^{*}-\left(t+\frac{q(t)}{s}\right)\right], \text { for } t \in\left[t_{s}, t_{o}\right], \\
\alpha \frac{q(t)}{s}+\gamma\left[\left(t+\frac{q(t)}{s}\right)-t^{*}\right], \text { for } t \in\left[t_{o}, t_{e}\right]
\end{array}\right.
$$

The equilibrium flow pattern after a fluctuation, the corresponding queue length, the travel time and the generalized travel cost are given as follows:

$$
\tilde{q}(t)=\left\{\begin{array}{l}
\left(\frac{\beta}{\alpha-\beta} s+\Delta_{1}\right)\left(t-t_{s}\right), \text { for } t \in\left[t_{s}, t_{o}\right], \\
\left(\frac{\beta}{\alpha-\beta} s+\Delta_{1}\right)\left(t_{o}-t_{s}\right)+\left(\frac{-\gamma}{\alpha+\gamma} s+\Delta_{2}\right)\left(t-t_{o}\right), \text { for } t \in\left[t_{o}, t_{e}\right],
\end{array}\right.
$$




$$
\begin{aligned}
& \tilde{T}(t)=\frac{\tilde{q}(t)}{s}=\left\{\begin{array}{l}
\left(\frac{\beta}{\alpha-\beta}+\frac{\Delta_{1}}{s}\right)\left(t-t_{s}\right), \text { for } t \in\left[t_{s}, t_{o}\right], \\
\left(\frac{\beta}{\alpha-\beta}+\frac{\Delta_{1}}{s}\right)\left(t_{o}-t_{s}\right)+\left(\frac{-\gamma}{\alpha+\gamma}+\frac{\Delta_{2}}{s}\right)\left(t-t_{o}\right), \text { for } t \in\left[t_{o}, t_{e}\right],
\end{array}\right. \\
& \tilde{C}(t)=\left\{\begin{array}{l}
\alpha \frac{\tilde{q}(t)}{s}+\beta\left[t^{*}-\left(t+\frac{\tilde{q}(t)}{s}\right)\right], \text { for } t \in\left[t_{s}, \tilde{t}_{o}\right], \\
\alpha \frac{\tilde{q}(t)}{s}+\gamma\left[\left(t+\frac{\tilde{q}(t)}{s}\right)-t^{*}\right], \text { for } t \in\left[\tilde{t}_{o}, t_{e}\right] .
\end{array}\right.
\end{aligned}
$$

where

$$
\tilde{t}_{0}=\frac{\alpha t_{0} s+\Delta_{1} t_{s}(\alpha-\beta)}{\alpha s+\Delta_{1}(\alpha-\beta)} .
$$

Using Eqs. (28), (75), (30) and (78), we have

$$
\begin{aligned}
\langle\tilde{\mathbf{C}}-\mathbf{C}, \tilde{\mathbf{r}}-\mathbf{r}\rangle= & \frac{\alpha}{s}\left\{\int_{t_{s}}^{t_{o}}[\tilde{q}(t)-q(t)] \Delta_{1} d t+\int_{t_{o}}^{t_{e}}[\tilde{q}(t)-q(t)] \Delta_{2} d t\right\} \\
& -\frac{\beta}{s} \int_{t_{s}}^{t_{0}^{\prime}}[\tilde{q}(t)-q(t)] \Delta_{1} d t+\frac{\gamma}{s} \int_{t_{o}}^{t_{e}}[\tilde{q}(t)-q(t)] \Delta_{2} d t \\
& +\int_{t_{o}^{\prime}}^{t_{o}}\left\{\gamma\left[\left(t+\frac{\tilde{q}(t)}{s}\right)-t^{*}\right]-\beta\left[t^{*}-\left(t+\frac{q(t)}{s}\right)\right]\right\} \Delta_{1} d t .
\end{aligned}
$$

Substituting Eqs. (73) and (76) into the first term on the right hand side of Eq. (80), we have

$$
\frac{\alpha}{s}\left\{\int_{t_{s}}^{t_{o}}[\tilde{q}(t)-q(t)] \Delta_{1} d t+\int_{t_{o}}^{t_{e}}[\tilde{q}(t)-q(t)] \Delta_{2} d t\right\}=0 .
$$

Substituting Eqs. (73), (76), (79) and (81) into Eq. (80), we have

$$
\langle\tilde{\mathbf{c}}-\mathbf{c}, \tilde{\mathbf{r}}-\mathbf{r}\rangle=-\frac{\alpha(\beta+\gamma) \Delta_{1}^{2}\left(t_{o}-t_{s}\right)^{2}}{2\left[\alpha s+\Delta_{1}(\alpha-\beta)\right]} .
$$

Let $\boldsymbol{\varphi}$ and $\tilde{\varphi}$, respectively, be the vectors of the perceived component of the perceived trip cost with respect to the flow patterns $\mathbf{r}$ and $\tilde{\mathbf{r}}$, i.e., $\boldsymbol{\varphi}=\left(\frac{1}{\theta_{t}} \ln r(t), \forall t\right)$ and $\tilde{\boldsymbol{\varphi}}=\left(\frac{1}{\theta_{t}} \ln \tilde{r}(t), \forall t\right)$. We have

$$
\begin{aligned}
& \langle\tilde{\boldsymbol{\varphi}}-\boldsymbol{\varphi}, \tilde{\mathbf{r}}-\mathbf{r}\rangle=\frac{1}{\theta_{t}}\left\{\int_{t_{s}}^{t_{o}}[\ln \tilde{r}(t)-\ln r(t)] \Delta_{1} d t+\int_{t_{o}}^{t_{e}}[\ln \tilde{r}(t)-\ln r(t)] \Delta_{2} d t\right\} \\
& =\frac{1}{\theta_{t}}\left\{\Delta_{1}\left(t_{o}-t_{s}\right) \ln \left[1+(\alpha-\beta) \Delta_{1} /(\alpha s)\right]+\Delta_{2}\left(t_{e}-t_{o}\right) \ln \left[1-(\alpha+\gamma) \Delta_{2} /(\alpha s)\right]\right\} \\
& =\frac{1}{\theta_{t}}\left\{\Delta_{1}\left(t_{o}-t_{s}\right) \ln \left[1+(\alpha-\beta) \Delta_{1} /(\alpha s)\right]+\Delta_{1}\left(t_{o}-t_{s}\right) \ln \left[1-(\alpha+\gamma) \Delta_{2} /(\alpha s)\right]\right\} \\
& =\frac{\Delta_{1}\left(t_{o}-t_{s}\right)}{\theta_{t}} \ln \left[\alpha s+(\alpha-\beta) \Delta_{1}\right]\left[\alpha s-(\alpha+\gamma) \Delta_{2}\right] .
\end{aligned}
$$


Hence, we have

$$
\begin{aligned}
& \langle\tilde{\hat{\mathbf{c}}}-\hat{\mathbf{c}}, \tilde{\mathbf{r}}-\mathbf{r}\rangle=\langle(\tilde{\mathbf{c}}+\tilde{\boldsymbol{\varphi}})-(\mathbf{c}+\boldsymbol{\varphi}), \tilde{\mathbf{r}}-\mathbf{r}\rangle=\langle\tilde{\mathbf{c}}-\mathbf{c}, \tilde{\mathbf{r}}-\mathbf{r}\rangle+\langle\tilde{\boldsymbol{\varphi}}-\boldsymbol{\varphi}, \tilde{\mathbf{r}}-\mathbf{r}\rangle \\
& =-\frac{\alpha(\beta+\gamma) \Delta_{1}^{2}\left(t_{o}-t_{s}\right)^{2}}{2\left[\alpha s+\Delta_{1}(\alpha-\beta)\right]}+\frac{\Delta_{1}\left(t_{o}-t_{s}\right)}{\theta_{t}} \ln \frac{\alpha s+\Delta_{1}(\alpha-\beta)}{\alpha s-\Delta_{1}(\alpha-\beta) \gamma / \beta} .
\end{aligned}
$$

\section{Appendix B}

The M-BFGS method proposed by Zhou and Li (2008) can be adopted to solve the nonlinear equations (46), and is outlined as follows:

Step 0. Initialization. Select an initial solution $\hat{\boldsymbol{\pi}}_{0}$, an initial symmetric positive definite matrix $\mathbf{H}_{0}$, the constant $\mu, \omega, \varpi \in(0,1)$, the convergence tolerances $\varepsilon_{2}, \varepsilon_{3}>0$, and set $\imath=0$.

Step 1. Checking stopping criterion. If $G_{3}\left(\hat{\boldsymbol{\pi}}_{l}\right)<\varepsilon_{2}$, then terminates the algorithm.

Step 2. Determination of the descent direction and stepsize. Obtain the descent direction $\mathbf{d}_{t}=-\mathbf{H}_{t} \mathbf{Z}\left(\hat{\boldsymbol{\pi}}_{l}\right)$. Set $i_{t}$ be the smallest nonnegative integer $i$ such that

$$
-\mathbf{Z}\left(\hat{\boldsymbol{\pi}}_{t}+\omega^{i} \mathbf{d}_{t}\right)^{\mathrm{T}} \mathbf{d}_{t} \geq \sigma \omega^{i}\left\|\mathbf{Z}\left(\hat{\boldsymbol{\pi}}_{t}+\omega^{i} \mathbf{d}_{t}\right)\right\|\left\|\mathbf{d}_{t}\right\|^{2} .
$$

Set $\lambda_{t}=\omega^{i_{t}}$ and $\mathbf{v}_{t}=\hat{\boldsymbol{\pi}}_{t}+\lambda_{t} \mathbf{d}_{t}$. If $\left\|\mathbf{Z}\left(\mathbf{v}_{t}\right)\right\| \leq \varepsilon_{3}$, then stop the algorithm; Otherwise, go to Step 3.

Step 3. Update of the minimum generalized travel cost. Compute

$$
\hat{\boldsymbol{\pi}}_{t+1}=\hat{\boldsymbol{\pi}}_{t}+\frac{\mathbf{Z}\left(\mathbf{v}_{l}\right)^{\mathrm{T}}\left(\hat{\boldsymbol{\pi}}_{t}-\mathbf{v}_{l}\right)}{\left\|\mathbf{Z}\left(\mathbf{v}_{l}\right)\right\|^{2}} \mathbf{Z}\left(\mathbf{v}_{l}\right) .
$$

Step 4. Update of the symmetric positive definite matrix. Compute $\mathbf{H}_{t+1}$ by the following update process

$$
\mathbf{H}_{t+1}=\mathbf{H}_{l}+\frac{\left(\mathbf{s}_{l}^{T} \mathbf{y}_{l}+\mathbf{y}_{l}^{T} \mathbf{H}_{l} \mathbf{y}_{l}\right)\left(\mathbf{s}_{l} \mathbf{s}_{l}^{T}\right)}{\left(\mathbf{s}_{l}^{T} \mathbf{y}_{l}\right)^{2}}-\frac{\mathbf{H}_{l} \mathbf{y}_{l} \mathbf{s}_{l}^{T}+\mathbf{s}_{l} \mathbf{y}_{l}^{T} \mathbf{H}_{l}}{\mathbf{s}_{l}^{T} \mathbf{y}_{l}} ;
$$

where,

$$
\mathbf{s}_{t}=\mathbf{v}_{t}-\hat{\boldsymbol{\pi}}_{t} \text {, and } \mathbf{y}_{t}=\mathbf{Z}\left(\mathbf{v}_{t}\right)-\mathbf{Z}\left(\hat{\boldsymbol{\pi}}_{t}\right)+\varpi \mathbf{s}_{l}
$$

Set $l=\imath+1$, and return to Step 1 .

Similar to the BFGS method presented in Subsection 4.3.1, we also use the iterative method to obtain the initial solution $\hat{\boldsymbol{\pi}}_{0}$, and set the initial symmetric positive definite matrix $\mathbf{H}_{0}$ be an identity matrix in Step 0. The major differences in terms of procedure between the M-BFGS and the BFGS methods are: (i) different line search methods are used in Step 2, and (ii) different methods to update the minimum generalized travel cost in Step 3. The convergence requirement of the M-BFGS method is weaker than that of the BFGS method. The M-BFGS method has been proved to converge globally if the nonlinear equation is monotone and Lipschitz continuous without the differentiability 
requirement on the equation, which makes it possible to solve some nonsmooth equations (Zhou and Li, 2008).

\section{References}

Anand, A., Ramadurai, G., Vanajakshi, L., 2014. Data fusion-based traffic density estimation and prediction. Journal of Intelligent Transportation Systems, 18(4), 367-378.

Arnott, R., de Palma, A., Lindsey, R., 1990. Departure time and route choice for the morning commute. Transportation Research Part B, 24 (3), 209-228.

Balijepalli, N.C., Ngoduy, D., Watling, D.P., 2014. The two-regime transmission model for network loading in dynamic traffic assignment problems. Transportmetrica A, 10 (7), 563-584.

Ban, X., Liu, H.X., Ferris, M.C., Ran, B., 2008. A link-node complementarity model and solution algorithm for dynamic user equilibria with exact flow propagations. Transportation Research Part B, 42 (9), 823-842.

Ban, X., Pang, J.S., Liu, H.X., Ma, R., 2012a. Continuous-time point-queue models in dynamic network loading. Transportation Research Part B, 46 (3), 360-380.

Ban, X., Pang, J.S., Liu, H.X., Ma, R., 2012b. Modeling and solving continuous-time instantaneous dynamic user equilibria: A differential complementarity systems approach. Transportation Research Part B, 46, 389-408.

Bekhor, S., Toledo, B., 2005. Investigating path-based solution algorithms to the stochastic user equilibrium problem. Transportation Research Part B, 39 (3), 279-295.

Blumberg-Nitzani, M., Bar-Gera, H., 2014. The effect of signalised intersections on dynamic traffic assignment solution stability. Transportmetrica A, 10(7), 622-646.

Carey, M., Ge, Y.E., 2012. Comparison of methods for path inflow reassignment for dynamic user equilibrium. Networks and Spatial Economics, 12(3), 337-376.

Carey, M., Srinivasan, A., 1993. Externalities, average and marginal costs, and tolls on congested networks with time-varying flows. Operations Research, 41 (1), 217-231.

Carey, M., Subrahmanian, E., 2000. An approach to modeling time-varying flows on congested networks. Transportation Research Part B, 34 (3), 157-183.

Carey, M., Watling, D., 2012. Dynamic traffic assignment approximating the kinematic wave model: system optimum, marginal costs, externalities and tolls. Transportation Research Part B, 46 (5), 634-648.

Chen, H.K., 1999. Dynamic travel choice models: a variational inequality approach. Springer, Berlin, Germany.

Chen, H.K., Feng, G., 2000. Heuristics for the stochastic/dynamic user-optimal route choice problem. 
European Journal of Operational Research, 126(1), 13-30.

Chen, X., Li, Z., Li, L., Shi, Q., 2014. Characterising scattering features in flow-density plots using a stochastic platoon model. Transportmetrica A, 10(9), 820-848.

Chiou, Y.-C., Lan, L.W., Tseng, C.-M., 2014. A novel method to predict traffic features based on rolling self-structured traffic patterns. Journal of Intelligent Transportation Systems, 18(4), 352-366.

Chiabaut, N., Xie, X., Leclercq, L., 2014. Performance analysis for different designs of a multimodal urban arterial. Transportmetrica B, 2(3), 229-245.

Chow, A.H.F., 2009. Dynamic system optimal traffic assignment - a state-dependent control theoretic approach. Transportmetrica, 5(2), 85-106.

Daganzo, C.F., 1995. The cell transmission model, Part II: Network traffic. Transportation Research Part B, 29 (2), 79-93.

Daganzo, C.F., Sheffi, Y., 1977. On stochastic models of traffic assignment. Transportation Science, 11 (3), 253-274.

Dial, R.B., 1971. A probabilistic multipath traffic assignment model which obviates path enumeration. Transportation Research, 5 (2), 83-111.

Friesz, T.L., Bernstein, D., Smith, T.E., Tobin, R.L., Wie, B., 1993. A variational inequality formulation of the dynamic networks user equilibrium problem. Operations Research, 41 (1), 179-191.

Friesz, T.L., Meimand, A., 2014. A differential variational inequality formulation of dynamic network user equilibrium with elastic demand. Transportmetrica A, 10(7), 661-668.

Ghali, M.O., Smith, M.J., 1993. Traffic assignment, traffic control and road pricing. In: Daganzo, C.F. (Ed.), Transportation and Traffic Theory. Elsevier, Amsterdam, The Netherlands, pp. 147-169.

Ghosh, B., Smith, D.P., 2014. Customization of automatic incident detection algorithms for signalized urban arterials. Journal of Intelligent Transportation Systems, 18(4), 426-441.

Han, K., Friesz, T.L., Yao, T., 2013a. A partial differential equation formulation of Vickrey's bottleneck model, part II: Numerical analysis and computation. Transportation Research Part B, 49, 75-93.

Han, K., Friesz, T.L., Yao, T., 2013b. Existence of simultaneous route and departure choice dynamic user equilibrium. Transportation Research Part B, 53, 17-30.

Han, S., 2003. Dynamic traffic modelling and dynamic stochastic user equilibrium assignment for general road networks. Transportation Research Part B, 37 (3), 225-249.

Heydecker, B. G., Addison, J. D., 2005. Analysis of dynamic traffic equilibrium with departure time choice. Transportation Science, 39(1), 39-57. 
Huang, H.J., Lam, W.H.K., 2002. Modeling and solving the dynamic user equilibrium route and departure time choice problem in network with queues. Transportation Research Part B, 36 (3), 253-273.

Iryo, T., 2013. Properties of dynamic user equilibrium solution: existence, uniqueness, stability, and robust solution methodology. Transportmetrica B, 1(1), 52-67.

Jang, W., Ran, B., Choi, K., 2005. A discrete time dynamic flow model and a formulation and solution method for dynamic route choice. Transportation Research Part B 39 (7), 593-620.

Johnson, C. R., 1970. Positive definite matrices. The American Mathematical Monthly, 77 (3), 259-264.

Leblanc, L.J., 1975. An algorithm for the discrete network design problem. Transportation Science, 9 (3), 183-199.

Lim, Y., Heydecker, B., 2005. Dynamic departure time and stochastic user equilibrium assignment. Transportation Research Part B, 39(2), 97-118.

Lindsey, R., 2004. Existence, uniqueness, and trip cost function properties of user equilibrium in the bottleneck model with multiple user classes. Transportation Science, 38 (3), 293-314.

Liu, H., He, X., He, B., 2009. Method of successive weighted averages (MSWA) and self-regulated averaging schemes for solving stochastic user equilibrium problem. Networks and Spatial Economics, 9 (4), 485-503.

Lo, H.K., Szeto, W.Y., 2002a. A cell-based variational inequality formulation of the dynamic user optimal assignment problem. Transportation Research Part B, 36 (5), 421-443.

Lo, H.K., Szeto, W.Y., 2002b. A cell-based dynamic traffic assignment model: formulation and properties. Mathematical and Computer Modelling, 35(7-8), 849-865.

Lo, H.K., Szeto, W.Y., 2005. Road pricing modeling for hyper- congestion. Transportation Research Part A, 39(7-9), 705-722.

Long, J.C., Gao, Z.Y., Zhang, H.Z., Szeto, W.Y., 2010, A turning restriction design problem in urban road networks. European Journal of Operational Research, 206 (3), 569-578.

Long, J.C., Huang H.J., Gao, Z.Y., Szeto, W.Y., 2013. An intersection-movement-based dynamic user optimal route choice problem. Operations Research, 61 (5), 1134-1147.

Ma, R., Ban, X., Pang, J.-S., 2014. Continuous-time dynamic system optimum for single-destination traffic networks with queue spillbacks. Transportation Research Part B, 68, 98-122.

Mahmassani, H., Herman, R., 1984. Dynamic user equilibrium departure time and route choice on idealized traffic arterials. Transportation Science, 18 (4), 362-384.

Meng, Q., Khoo, H.L. 2012. A computational model for the probit-based dynamic stochastic user optimal traffic assignment problem. Journal of Advanced Transportation, 46 (1), 80-94. 
Merchant, D.K, Nemhauser, G.L., 1978a. A model and an algorithm for the dynamic traffic assignment. Transportation Science, 12 (3), 183-199.

Merchant, D.K, Nemhauser, G.L., 1978b. Optimality conditions for a dynamic traffic assignment model. Transportation Science, 12 (3), 200-207.

Mesa-Arango, R., Ukkusuri, S.V., 2014. Modeling the car-truck interaction in a system-optimal dynamic traffic assignment model. Journal of Intelligent Transportation Systems, 18(4), 327-338.

Miandoabchi, E., Farahani, R.Z., Szeto, W.Y., 2012a. Bi-objective bimodal urban road network design using hybrid metaheuristics. Central European Journal of Operations Research, 20(4), 583-621.

Miandoabchi, E., Farahani, R.Z., Dullaert, W., Szeto, W.Y., 2012b. Hybrid evolutionary metaheuristics for concurrent multi-objective design of urban road and public transit networks. Networks and Spatial Economics, 12(3), 441-480.

Mounce, R., Carey, M., 2011. Route swapping in dynamic traffic networks. Transportation Research Part B, 45 (1), 102-111.

Mun, J.S., 2007. Traffic performance models for dynamic traffic assignment: An assessment of existing models, Transport Reviews, 27 (2), 231-249.

Mun, J.S., 2011. A solution algorithm for a dynamic deterministic user equilibrium assignment model with departure time choice. Transportation Planning and Technology, 34 (5), 443-466.

Nagurney, A., 1993. Network Economics: A Variational Inequality Approach. Kluwer Academic Publishers, Norwell, Massachusetts, USA.

Ngoduy, D., 2013. Platoon-based macroscopic model for intelligent traffic flow. Transportmetrica B, 1(2), 153-169.

Nie, X., Zhang, H., 2005. A comparative study of some macroscopic link models used in dynamic traffic assignment. Networks and Spatial Economics, 5 (1), 89-115.

Nie, Y., 2011. A cell-based Merchant-Nemhauser model for the system optimum dynamic traffic assignment problem. Transportation Research Part B, 45 (2), 329-342.

Nie, Y., Zhang, H.M. 2010. Solving the dynamic user optimal assignment problem considering queue spillback. Networks and Spatial Economics, 10, 49-71.

Panicucci, B., Pappalardo, M., Passacantando, M., 2007. A path-based double projection method for solving the asymmetric traffic network equilibrium problem. Optimization Letters, 1 (2), 171-185.

Qian, Z., Zhang, H.M., 2013. The morning commute problem with heterogeneous travellers: the case of continuously distributed parameters. Transportmetrica A, 9(2), 178-203.

Ran, B., Boyce, D.E., 1996. Modeling Dynamic Transportation Network: An Intelligent Transportation System Oriented Approach. Springer, Heidelberg.

Ran, B., Lee, D.-H., Shin, M.S.-I., 2002. New algorithm for a multiclass dynamic traffic assignment 
model. Journal of Transportation Engineering, 128, 323-335.

Shen, W., Zhang, H.M., 2014. System optimal dynamic traffic assignment: Properties and solution procedures in the case of a many-to-one network. Transportation Research Part B, 65, 1-17.

Siu, B.W.Y., Lo, H.K., 2013. Punctuality-based departure time scheduling under stochastic bottleneck capacity: formulation and equilibrium. Transportmetrica B, 1(3), 195-225.

Siu, B.W.Y., Lo, H.K., 2014. Punctuality-based route and departure time choice. Transportmetrica A, 10(7), 585-621.

Small, K., 1982. The scheduling of consumer activities: work trips. American Economics Review, 72 (3), 467-479.

Smith, M.J., Ghali, M.O., 1990. Dynamic traffic assignment and dynamic traffic control. In: Proceedings of the 11th International Symposium on Transportation and Traffic Theory, pp. 273-290.

Solodov, M.V., Svaiter, B.F., 1998. A globally convergent inexact Newton method for systems of monotone equations. In: M. Fukushima, L. Qi (Eds.), Reformulation: Nonsmooth, Piecewise smooth, Semismooth and Smoothing Methods, Kluwer Academic Publishers, pp. 355-369.

Sumalee, A., Zhong, R.X., Pan, T.L., Szeto, W.Y., 2011. Stochastic cell transmission model (SCTM): a stochastic dynamic traffic model for traffic state surveillance and assignment. Transportation Research Part B, 45(3), 507-533.

Szeto, W.Y., 2008. The enhanced lagged cell transmission model for dynamic traffic assignment. Transportation Research Record, 2085, 76-85.

Szeto, W.Y., Ghosh, B., Basu, B., O’Mahony, M., 2009. Cell-based short-term traffic flow forecasting using time series modelling. ASCE Journal of Transportation Engineering, 135(9), 658-667.

Szeto, W.Y., Jaber, X.Q., O’Mahony, M., 2010. Time-dependent discrete network design frameworks considering land use. Computer-Aided Civil and Infrastructure Engineering, 25(6), 411-426.

Szeto, W.Y., Jiang, Y., Sumalee, A., 2011. A cell-based model for multi-class doubly stochastic dynamic traffic assignment. Computer-Aided Civil and Infrastructure Engineering, 26 (8), 595-611.

Szeto, W.Y., Jiang, Y., Wang, D.Z.W., Sumalee, A., 2014. A sustainable road network design problem with land use transportation interaction over time. Networks and Spatial Economics, DOI: 10.1007/s11067-013-9191-9.

Szeto, W.Y., Lo, H.K., 2004. A cell-based simultaneous route and departure time choice model with elastic demand. Transportation Research Part B, 38 (7), 593-612.

Szeto, W.Y., Lo, H.K., 2006. Dynamic traffic assignment: Properties and extensions. Transportmetrica, 2 (1), 31-52. 
Szeto, W.Y., Wong, S.C., 2012. Dynamic traffic assignment: model classifications and recent advances in travel choice principles. Central European Journal of Engineering, 2 (1), 1-18.

Tian, L.J., Huang, H.J., Gao, Z.Y., 2012. A cumulative perceived value-based dynamic user equilibrium model considering the travelers' risk evaluation on arrival time. Networks and Spatial Economics, 12 (4), 589-608.

Vickrey, W.S., 1969. Congestion theory and transport investment. American Economic Review, 59 (2), 251-261.

Vythoulkas, P., 1990. A dynamic stochastic assignment model for the analysis of general networks. Transportation Research Part B, 24 (6), 453-469.

Williams, H.C.W.L., 1977. On the formation of travel demand models and economic evaluation measures of user benefit. Environment and Planning A, 9 (3), 285-344.

Wu, J.H., Florian, M., Xu, Y.W., Rubio-Ardanaz, J.M., 1998. A projection algorithm for the dynamic network equilibrium problem. In: Proceedings of 1998 International Conference on Traffic and Transportation Studies. American Society of Civil Engineers, Beijing, pp. 379-390.

Ye, Q., Szeto, W.Y., Wong, S.C., 2012. Short-term traffic speed forecasting based on data recorded at irregular intervals. IEEE Transactions on Intelligent Transportation Systems, 13(4), 1727-1737.

Yperman, I., 2007. The link transmission model for dynamic network loading. Ph D. dissertation, Katholieke Universiteit Leuven, Leuven, Belgium.

Yuan, G., Lu, X., 2008. A new back tracking inexact BFGS method for symmetric nonlinear equations. Computers \& Mathematics with Applications, 55 (1), 116-129.

Yushimito, W.F., Ban, X., Holguín-Veras, J., 2014. A two-stage optimization model for staggered work hours. Journal of Intelligent Transportation Systems, 18(4), 410-425.

Zhang, D., Nagurney, A., Wu, J.H., 2001. On the equivalence between stationary link flow patterns and traffic network equilibria. Transportation Research Part B, 35, 731-748.

Zhang, H.M., Nie, Y., Qian, Z., 2013. Modelling network flow with and without link interactions: the cases of point queue, spatial queue and cell transmission model. Transportmetrica B, 1(1), 33-51.

Zhao, Y.B., Hu, J., 2007. Global bounds for the distance to solutions of co-coercive variational inequalities. Operations Research Letters, 35 (3), 409-415.

Zheng, H., Chang, Y.C., 2011. A network flow algorithm for the cell-based single-destination system optimal dynamic traffic assignment problem. Transportation Science, 45 (1), 121-137.

Zhou, W.J., Li, D.H., 2008. A globally convergent BFGS method for nonlinear monotone equations without any merit functions. Mathematics of Computation, 77 (264), 2231-2240.

Zhong, R.X., Sumalee, A., Pan, T.L., Lam, W.H.K., 2013. Stochastic cell transmission model for traffic network with demand and supply uncertainties. Transportmetrica A, 9(7), 567-602. 
Zhu, F., Lo, H.K., Lin, H.-Z., 2013. Delay and emissions modelling for signalised intersections. Transportmetrica B, 1(2), 111-135.

Zhu, F., Ukkusuri, S.V., 2013. A cell based dynamic system optimum model with non-holding back flows. Transportation Research Part C, 36, 367-380.

Ziliaskopoulos, A.K., 2000. A linear programming model for the single destination system optimum dynamic traffic assignment problem. Transportation Science, 34 (1), 37-44. 
Table 1.

Reasonable path set for each OD pair.

\begin{tabular}{cccccc}
\hline OD pair & Number of paths & OD pair & Number of paths & OD pair & Number of paths \\
\hline $1->10$ & 6 & $12->19$ & 6 & $20->9$ & 7 \\
$4->19$ & 8 & $13->10$ & 5 & $22->8$ & 6 \\
$6->15$ & 4 & $14->8$ & 8 & $2->15$ & 4 \\
$7->15$ & 6 & $18->5$ & 4 & $3->16$ & 9 \\
\hline
\end{tabular}

Table 2.

The effect of dispersion parameters on the number of DNLs required for each solution algorithm.

\begin{tabular}{ccccccc}
\hline \multirow{2}{*}{$\theta_{r}$ and $\theta_{t}\left(\mathrm{~s}^{-1}\right)$} & \multicolumn{7}{c}{ Number of DNLs required } \\
\cline { 2 - 7 } & PNL & MSA & SRAM & IM & M-BFGS & BFGS \\
\hline 0.05 & 9 & 513 & 20 & 43 & 43 & 43 \\
0.1 & - & 20778 & 51 & 100 & 101 & 101 \\
0.15 & - & 163590 & 176 & 178 & 177 & 177 \\
0.2 & - & - & 900 & 267 & 265 & 265 \\
0.25 & - & - & 14531 & 462 & 461 & 461 \\
0.3 & - & - & 74343 & 731 & 731 & 731 \\
0.35 & - & - & - & 1174 & 1174 & 1174 \\
0.4 & - & - & - & 1742 & 1742 & 1742 \\
0.45 & - & - & - & 2653 & 2653 & 2653 \\
0.5 & - & - & - & 32073 & 51364 & 14964 \\
0.55 & - & - & - & - & 55392 & 20562 \\
0.6 & - & - & - & - & 83785 & 22647 \\
0.65 & - & - & - & - & 68666 & 25681 \\
0.7 & - & - & - & - & 171750 & 25709 \\
0.75 & - & - & - & - & 164865 & 27834 \\
\hline
\end{tabular}


Table 3.

The effect of the dispersion parameter $\theta_{t}$ on the number of DNLs required for each solution algorithm $\left(\theta_{r}=1.00 \mathrm{~s}^{-1}\right)$.

\begin{tabular}{ccccccc}
\hline \multirow{2}{*}{$\theta_{t}\left(\mathrm{~s}^{-1}\right)$} & \multicolumn{7}{c}{ Number of DNLs required } \\
\cline { 2 - 7 } & PNL & MSA & SRAM & IM & M-BFGS & BFGS \\
\hline 0.05 & - & 51872 & 42 & 943 & 943 & 943 \\
0.10 & - & 157186 & 104 & 956 & 956 & 956 \\
0.15 & - & - & 209 & 986 & 986 & 986 \\
0.20 & - & - & 479 & 976 & 976 & 976 \\
0.25 & - & - & 4905 & 994 & 994 & 994 \\
0.30 & - & - & - & 1055 & 1055 & 1055 \\
0.35 & - & - & - & 1627 & 1627 & 1627 \\
0.40 & - & - & - & 4622 & 4622 & 4622 \\
0.45 & - & - & - & - & 98610 & 35996 \\
0.50 & - & - & - & - & 95268 & 38307 \\
0.55 & - & - & - & - & 105098 & 36651 \\
0.60 & - & - & - & - & 127071 & 36273 \\
0.65 & - & - & - & - & 335945 & 36988 \\
0.70 & - & - & - & - & 232170 & 35388 \\
0.75 & - & - & - & - & 125743 & 37283 \\
0.80 & - & - & - & - & 111014 & 42083 \\
0.85 & - & - & - & - & 429875 & 41201 \\
0.90 & - & - & - & - & 166203 & 40904 \\
0.95 & - & - & - & - & 112805 & 43074 \\
1.00 & - & - & - & 96272 & 38185 \\
\hline
\end{tabular}


Table 4.

The effect of traffic demand on the number of DNLs required for each solution algorithm $\left(\theta_{r}=\theta_{t}=0.50 \mathrm{~s}^{-1}\right)$.

\begin{tabular}{ccccccc}
\hline \multirow{2}{*}{ Demand } & \multicolumn{7}{c}{ Number of DNLs required } \\
\cline { 2 - 7 } & PNL & MSA & SRAM & IM & M-BFGS & BFGS \\
\hline 20 & 2 & 2 & 19 & 495 & 495 & 495 \\
40 & 2 & 2 & 21 & 529 & 529 & 529 \\
60 & - & 3617 & 48 & 552 & 552 & 552 \\
80 & - & 237734 & 732 & 632 & 632 & 632 \\
100 & - & - & 9296 & 755 & 755 & 755 \\
120 & - & - & 94491 & 909 & 909 & 909 \\
140 & - & - & - & 1387 & 1387 & 1387 \\
160 & - & - & - & 1412 & 1412 & 1412 \\
180 & - & - & - & 1975 & 1975 & 1975 \\
200 & - & - & - & 32033 & 51364 & 14964 \\
220 & - & - & - & - & 132134 & 18005 \\
240 & - & - & - & - & 96137 & 18630 \\
260 & - & - & - & - & 73938 & 18203 \\
280 & - & - & - & - & 48072 & 18569 \\
300 & - & - & - & - & 47231 & 17890 \\
320 & - & - & - & - & 54131 & 17803 \\
340 & - & - & - & - & 50905 & 15241 \\
360 & - & - & - & - & 63463 & 16313 \\
380 & - & - & - & - & 203539 & 14406 \\
400 & - & - & - & &
\end{tabular}

Table 5.

The effect of $\gamma$ on the number of DNLs required for each solution algorithm $\left(\theta_{r}=\theta_{t}=1.00 \mathrm{~s}^{-1}\right)$.

\begin{tabular}{ccccccc}
\hline & \multicolumn{6}{c}{ Number of DNLs required } \\
\cline { 2 - 7 } & PNL & MSA & SRAM & IM & M-BFGS & BFGS \\
\hline 1.0 & - & - & - & 2324 & 2324 & 2324 \\
1.2 & - & - & - & 4765 & 40718 & 9857 \\
1.4 & - & - & - & 7647 & 47325 & 11880 \\
1.6 & - & - & - & - & 53264 & 17608 \\
1.8 & - & - & - & - & 55603 & 19309 \\
2.0 & - & - & - & - & 64214 & 21032 \\
2.2 & - & - & - & - & 68864 & 22732 \\
2.4 & - & - & - & - & 78117 & 24085 \\
2.6 & - & - & - & - & 74363 & 27880 \\
2.8 & - & - & - & - & 79031 & 27296 \\
3.0 & - & - & - & - & 88794 & 30488 \\
\hline
\end{tabular}




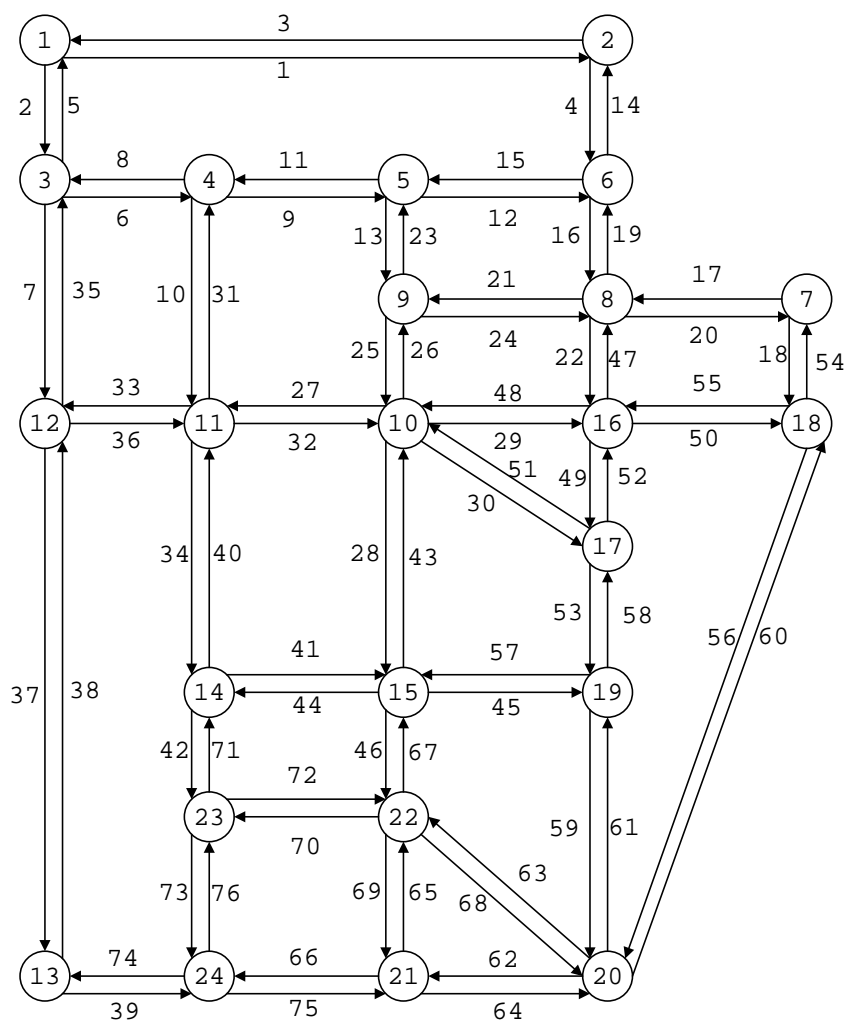

Fig. 1. The Sioux Falls network.

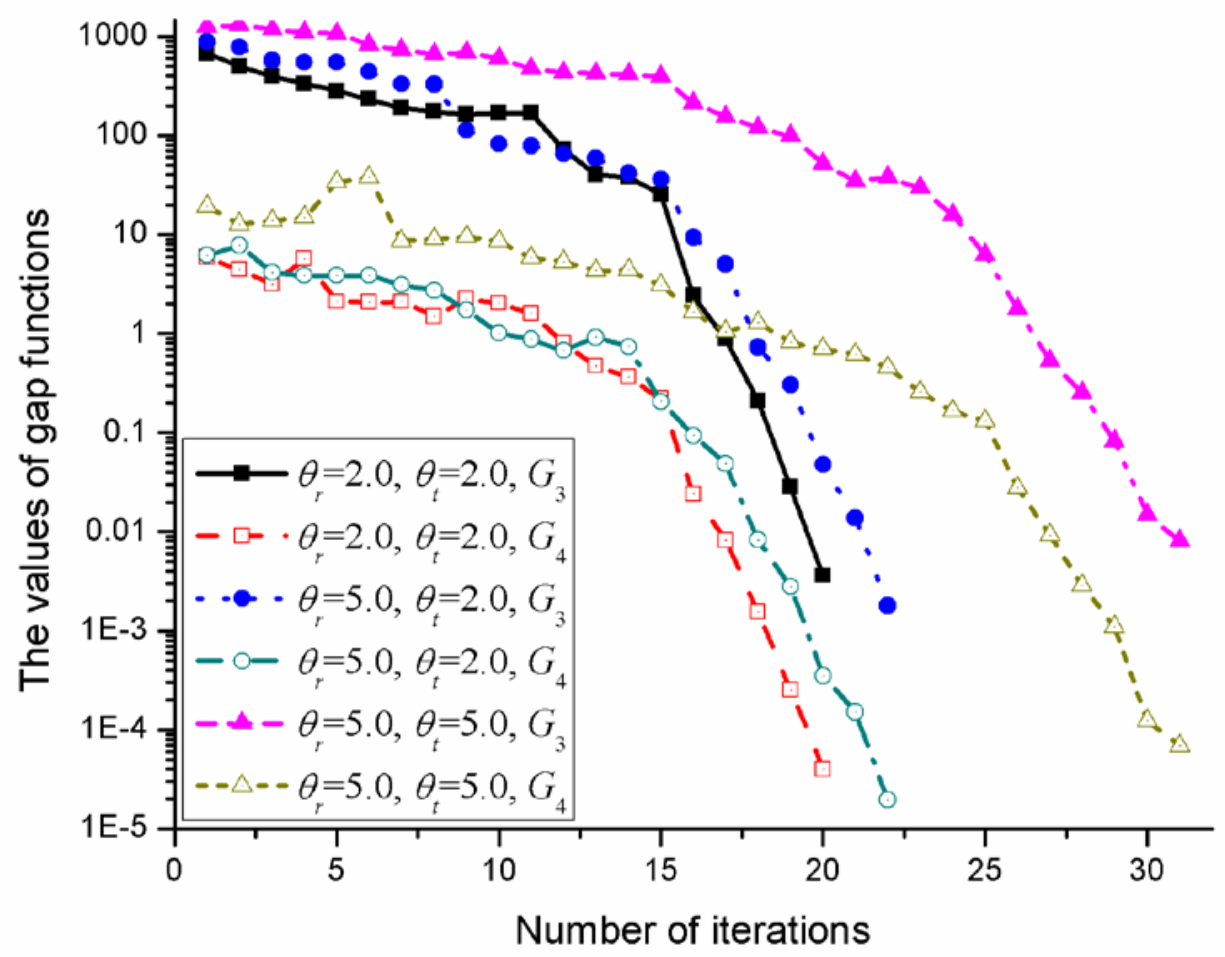

Fig. 2. Convergence of the BFGS method for solving the DSUE-SRDTC problem with high values of dispersion parameters. 


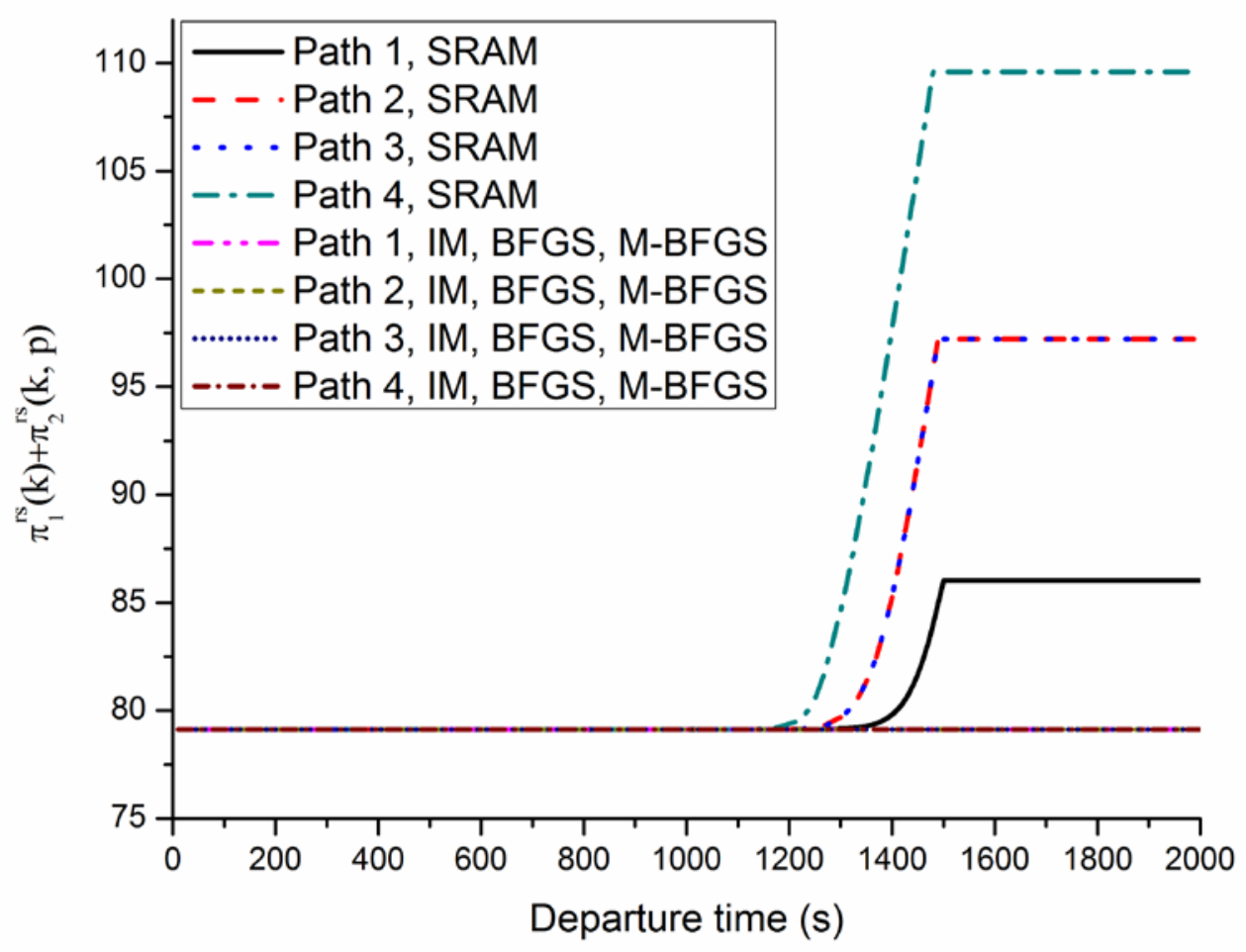

Fig. 3. The values of $\pi_{1}^{r s}(k)+\pi_{2}^{r s}(k, p)$ with respect to OD pair 6-> 15 . when $\theta_{r}=\theta_{t}=0.2 \mathrm{~s}^{-1}$.

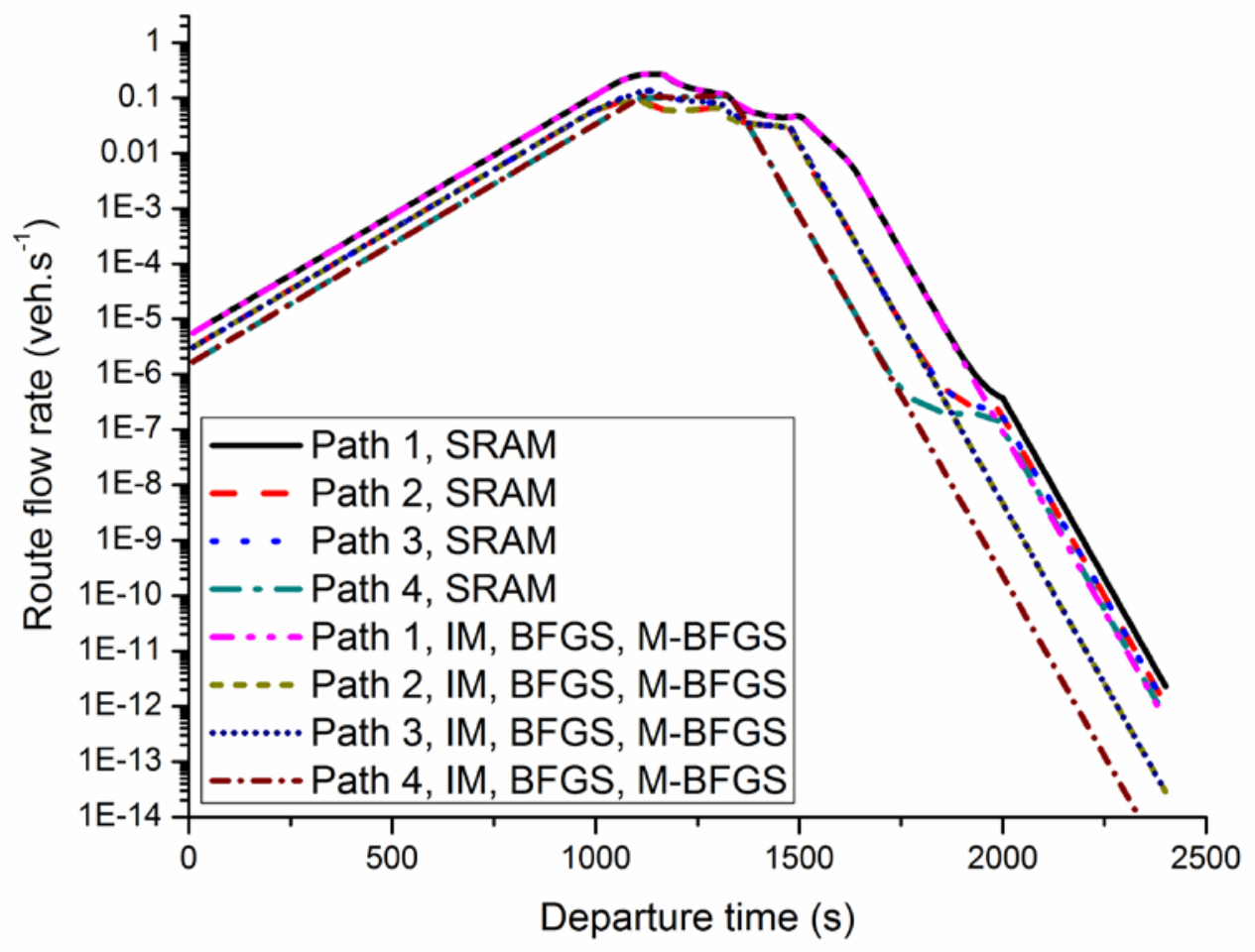

Fig. 4. Flow rates on routes between OD pair 6->15 obtained by different solution algorithms

$$
\left(\theta_{r}=\theta_{t}=0.2 \mathrm{~s}^{-1}\right) .
$$

